\title{
WestVirginiaUniversity
}

THE RESEARCH REPOSITORY @ WVU

Graduate Theses, Dissertations, and Problem Reports

2015

\section{Upscaling of the acidizing process in heterogeneous porous media}

Vahidreza Bitarafhaghighi

Follow this and additional works at: https://researchrepository.wvu.edu/etd

\section{Recommended Citation}

Bitarafhaghighi, Vahidreza, "Upscaling of the acidizing process in heterogeneous porous media" (2015). Graduate Theses, Dissertations, and Problem Reports. 5215.

https://researchrepository.wvu.edu/etd/5215

This Thesis is protected by copyright and/or related rights. It has been brought to you by the The Research Repository @ WVU with permission from the rights-holder(s). You are free to use this Thesis in any way that is permitted by the copyright and related rights legislation that applies to your use. For other uses you must obtain permission from the rights-holder(s) directly, unless additional rights are indicated by a Creative Commons license in the record and/ or on the work itself. This Thesis has been accepted for inclusion in WVU Graduate Theses, Dissertations, and Problem Reports collection by an authorized administrator of The Research Repository @ WVU. For more information, please contact researchrepository@mail.wvu.edu. 
Upscaling of the acidizing process in heterogeneous porous media

By

Vahidreza Bitarafhaghighi

Thesis submitted to the

Benjamin M. Statler College of Engineering and Mineral Resources

At West Virginia University

In partial fulfillment of the requirements

For the degree of

Master of Science

In

Petroleum and Natural Gas Engineering

Approved by

Ebrahim Fathi, PhD, Chair

Kashy Aminian, PhD

Fatemeh Belyadi, PhD.

Department of Petroleum and Natural Gas Engineering

Morgantown, West Virginia

2015

Keywords: Stochastic Modeling, Numerical Simulation, Acidizing, Heterogeneity Effect, Upscaling Model, Random fields

Copyright 2015 Vahidreza Bitarafhaghighi 


\title{
Abstract
}

\section{Upscaling of the acidizing process in heterogeneous porous media}

\author{
Vahidreza Bitarafhaghighi
}

Coupled fluid flow, reaction and transport in porous media has been the topic of research in various disciplines for the past few decades. Conventional approach assumes a homogeneous and isotropic porous media, and simplifies the nature of coupling between fluid and rock interactions. However, including the reality of the process, i.e. assuming heterogeneous and anisotropic porous media with fully coupled rock fluid interaction, can lead to more advanced understanding of the fundamental physics behind the problem and developing efficient industrial applications. In the oil and gas industry optimization of different well stimulation techniques such as matrix acidizing in order to enhance oil recovery requires an advanced understanding of fluid flow and also reaction in heterogeneous formations. This thesis is a contribution to development of more general governing equations describing the reactive flow and transport in heterogeneous formations.

The heterogeneity of the porous medium is introduced in the formulation through random permeability field that possess the characteristics of stationary stochastic process. The heterogeneity in permeability field affects the reservoir dynamics over a range of length and time scales by making pressure, concentration, diffusion and reaction coefficients stochastic random fields. Stochastic nature of these parameters helps us to be able to upscale the process while keeping the local information associated with heterogeneous nature of the porous media.

Conventional approaches to deal with this problem are homogenization and smoothing the heterogeneous properties of the formation using averaging based techniques such as up-gridding. However, these techniques do not carry the fundamental physics governing the process and cannot mimic the experimental observations such as acid front movement and instability of the reaction process. The local variations in rock and fluid properties are also ignored in these techniques which might lead to significant impacts in field scale application of acidizing as one of the major stimulation techniques.

In order to upscale the isothermal reaction process in a heterogeneous porous medium, according to the nature of the process, spectral-based small perturbation theory (Gelhar, 1993; Gelhar and Axness, 1983) is used among the various numerical and analytical upscaling techniques. The reaction is a nonlinear dissolution of an injected acid in a homogeneous liquid with constant density in a stationary mineral with constant porosity. In order to follow the acid front a moving coordinate is introduced. The upscaled governing equations are obtained with explicit macro-scale expressions for the coefficients and solved using time adaptive implicit finite difference technique. The results are compared with homogeneous models and sensitivity analysis of the upscaled equations is performed. Finally conclusions and results are discussed showing the importance of applying upscaling techniques to capture the impacts of heterogeneity on fluid dynamics. 


\section{Acknowledgements}

My deep love and gratitude go to my family. My parents were always there for me although being thousands of miles away. They supported me wholeheartedly and never let me feel alone. I am thankful to my brother, Ali, who always helped me to stay positive and advised me and to my sister, Shadi, who is the reason for my happiness.

I would like to thank Dr. Ebrahim Fathi for his guidance during the course of this study. My appreciation goes to Dr. Khashayar Aminian and Dr. Fatemeh Belyadi, who generously accepted to be a member of my thesis committee. They made significant contribution to this work and helped me to improve it.

I am especially thankful to Department Chair Professor Samuel Ameri for his unconditional support during the entire program. He always shared his knowledge and experience in a very helpful and friendly manner.

I would like to take the opportunity to thank Dr. Shahab Mohaghegh for being an inspiration all the time and for the time and effort he put in to teach me so many lessons. 


\section{Contents}

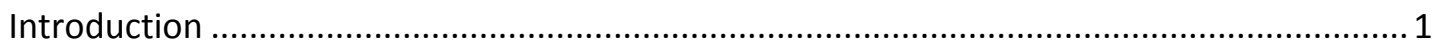

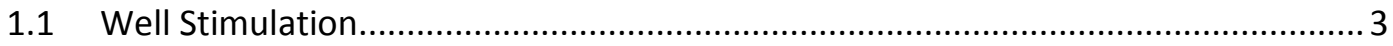

1.2 Transport in Heterogeneous media .................................................................. 4

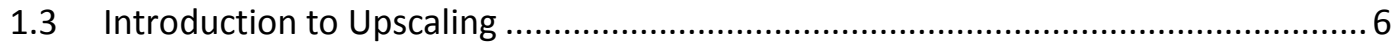

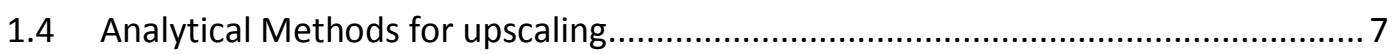

1.4.1 Integral Transform Methods .................................................................... 7

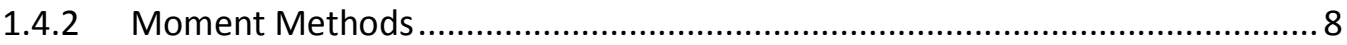

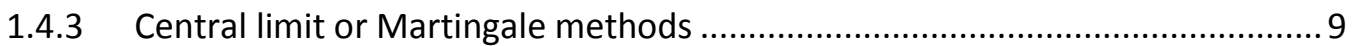

1.4.5 Mathematical homogenization .................................................................... 10

1.4.6 Mixture and hybrid mixture theory ………............................................ 11

1.4.7 Renormalization group techniques....................................................... 12

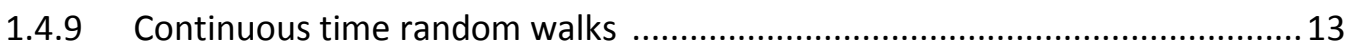

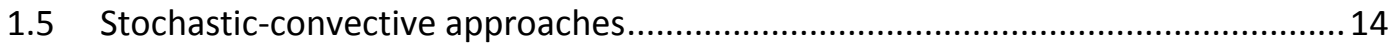

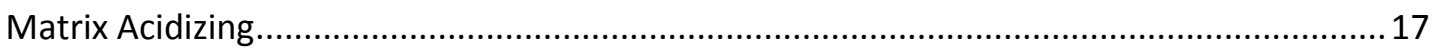

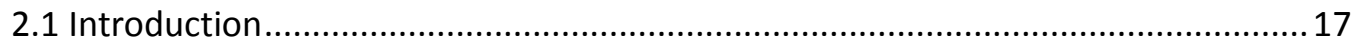

2.2Mathematical Model for Matrix Acidizing …….................................................... 19

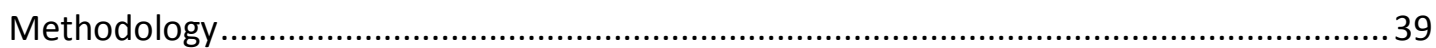

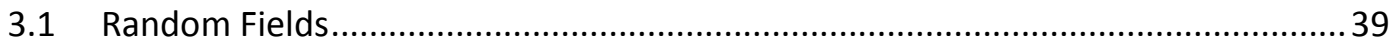

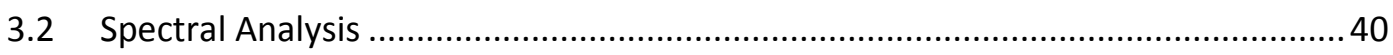

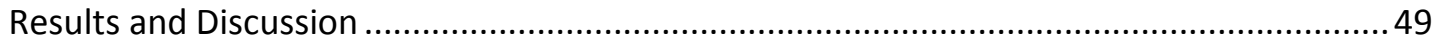

4.1 Field Scale Coefficients and Upscaled Reactive Flow System Dynamics ..................49

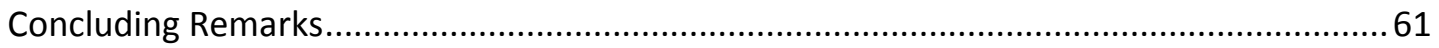

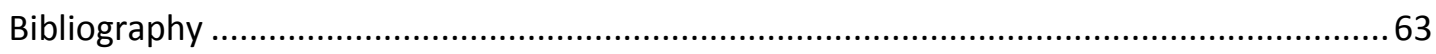

Appendix A - Estimation of the Reaction Wave Propagation Velocity.................................66

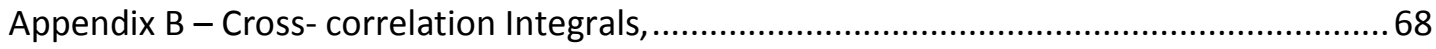




\section{List of Figures}

Figure 1. Schematic of constant pressure no flow boundary condition ..................................25

Figure 2. Early time effect of injection times on Acid concentration profiles .........................26

Figure 3. Early time effect of injection times on Mineral concentration profiles ....................2 27

Figure 4. Demonstration of acid concentration distribution in long injection times................28

Figure 5. acidizing effect on the mineral concentration in long times ....................................29

Figure 6. Effect of injection fluid acid concentration on Acid profile after 3h.......................30

Figure 7. Effect of injection fluid acid concentration on mineral profile after $3 \mathrm{~h} . . . \ldots \ldots \ldots \ldots \ldots . . . . .31$

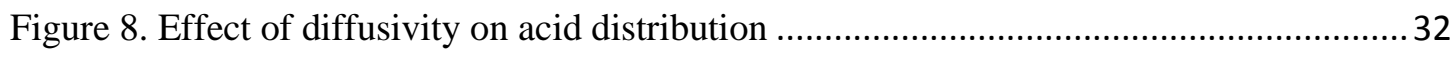

Figure 9. Effect of diffusivity on mineral distribution ......................................................... 33

Figure 10 . Impact of porosity on acid distribution............................................................ 34

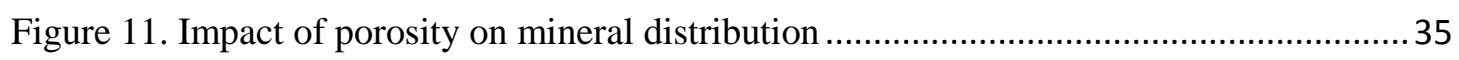

Figure 12. Acid distribution and different injecting acid linear velocities .............................36

Figure 13. Mineral distribution and different injecting acid linear velocities..........................37

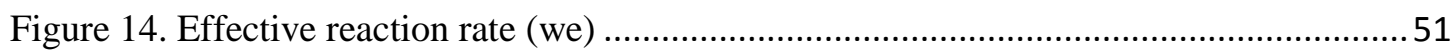

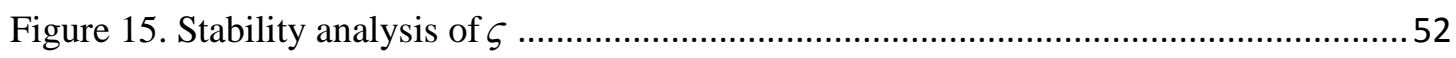

Figure 16. Effect of time on the Effective linear velocity (ve) ………................................53

Figure 17. Effect of permeability correlation length on the Effective linear velocity (ve) ......54

Figure 18. Effect of permeability variance on the Effective linear velocity (ve).....................55

Figure 19. Effect of time on the longitudinal effective dispersivity (A11) .............................57

Figure 20. Effect of permeability correlation length on effective dispersivity (A11)..............58

Figure 21. Effect of permeability variance on the effective dispersivity (A11) .....................59

Figure 22. Acid and mineral concentration in both Homogeneous and Heterogeneous cases 60 


\section{List of Tables}

Table 1. Parameters of Advection-Reaction Problem in Homogeneous Porous Media...........38

Table 2. Parameters of Advection-Reaction Problem in Heterogeneous Porous Media .........71 


\section{Nomenclature}

Y

$\eta$

$\omega$

$M_{t}$

$M_{m}$

$K_{i j}$

$\mu$

$D_{i j}$

$v_{i}$

$v$

$\tilde{v}$

$\rho_{m}$

$\rho$

$a_{s}$

W

$k_{0}$
Acid concentration

Mineral concentration

Reaction rate coefficient

Molecular weights of the tracer

Molecular weights of the mineral

Absolute permeability

Concentration-independent liquid viscosity

Tracer dispersion coefficient tensor in liquid phase

Linear velocity

mass-weighted stoichiometric coefficient for the tracer

Stoichiometric coefficient

Dissolvable mineral mass per total volume

Density of the carrier liquid

Mineral surface area

Mineral dissolution rate

Coefficient of reaction $\mathrm{mol} / \mathrm{ccP}$ ore

$\mathrm{mol} / \mathrm{ccSolid}$

$1 /(\mathrm{mol} / \mathrm{cc})^{*} \mathrm{t}$

md

cp

$\mathrm{cm}^{2} / \mathrm{s}$

$\mathrm{cm} / \mathrm{s}$ 


\begin{tabular}{|c|c|c|}
\hline$\alpha$ & Tracer reactant capacity & \\
\hline$\sigma_{f}$ & Standard deviation of $\ln \mathrm{K}$ & \\
\hline$\varnothing$ & Porosity & \\
\hline$\lambda_{i}$ & Permeability correlation length & $\mathrm{cm}$ \\
\hline$k_{i}$ & Wave number & \\
\hline$\delta$ & Correlation residual & \\
\hline$\alpha_{L}$ & local longitudinal dispersivity & $\mathrm{cm}^{2} / \mathrm{s}$ \\
\hline$\alpha_{T}$ & local transverse dispersivity & $\mathrm{cm}^{2} / \mathrm{s}$ \\
\hline$\delta_{i j}$ & The Kronecker delta function & \\
\hline $\bar{V}$ & Mean propagation speed of the reaction wave & $\mathrm{cm} / \mathrm{s}$ \\
\hline$v_{i}$ & Fluid velocity & $\mathrm{cm} / \mathrm{s}$ \\
\hline$\gamma$ & Flow factor & \\
\hline
\end{tabular}




\section{Chapter 1}

\section{Introduction}

The study of porous media has been around over a century. Henry Darcy was the first person who discovered the relationship between the flow rate and pressure (Darcy 1856). Performing some filtration experiments with a packed sand column, he empirically formulated that the flow rate is proportional to the balance of static and dynamic forces. This observation, known as Darcy's law, became vital in petroleum engineering and groundwater hydrology due to its significant applications such as in modeling the fluid flow in porous media in order to increase production and modify the treatment methods. It has been greatly used since its discovery. After a number of years, some theoretical studies were developed to justify Darcy's law to be applicable to the phenomena that happen in reality more commonly. The assumptions in Darcy's law such as homogeneous medium would limit this empirical equation to less practical cases. Stephen Whitaker made an enormous advancement by introducing the conception of finding equivalent descriptions for highly heterogeneous phenomena (Whitaker 1999). He analyzed the flow by averaging the pore scale description over a representative pore volume. His method has no prescribed rule on choosing a proper representative elementary volume, and it gained the attention of other scientists over time and encouraged them to find new techniques to obtain equivalent descriptions for the media characteristics in various contexts. Various numerical and analytical 
methods were developed to assess the effects of medium heterogeneity on fluid flow models.

In order to model the flow of fluid in the porous media, one will need to account for the spatial variability in the key parameters that affect the fluid flow, transport, storage, and reaction at a given scale. However, even if adequate computational resources are available, the amount of available data to determine the spatial distribution of the parameters with high certainty is not adequate to study the dynamics of fluid in heterogeneous formations. The challenge is how to incorporate the heterogeneous nature of porous media in governing equations describing the dynamics of fluid flow, storage, and reaction.

Heterogeneity affects the reservoir characteristic over a range of time and length scales. Depending on the nature of the problem, heterogeneity can impact convection, diffusion, and reaction in different time and length scales. Based on the length of the system, there are two major approaches to incorporate the heterogeneous rate-limited mass transfer into mathematical models for salute transport. One focuses on grain scale heterogeneity and the processes that operate at the microscopic scale, while the other one stresses the behavior of solute transport on the field scale and assesses the macroscopic variability of the medium.

This study upscales the process of fluid flow, transport and reaction in a porous media where the local heterogeneity in permeability field is considered. The analytically upscaled governing equations are then solved numerically to investigate the impact of permeability variations on transport and reaction parameters and quantify the acid front propagation in heterogeneous formation. 


\subsection{Well Stimulation}

Reservoir stimulation is a general term assigned to different techniques applied to increase the well productivity. A stimulation attempt first requires accurate identification of parameters that characterize the physical, chemical, and biological properties of natural porous media. These parameters control the well productivity.

Among the variables that affect the well productivity, permeability and skin effect are of particular interest to the stimulation engineer as they render post treatment analysis and job evaluations possible. Both of these variables can be obtained from a pressure transient well test. However, the lab scale values cannot be used throughout the whole reservoir and should be populated in a logistic way. This concept will be discussed thoroughly later in this study.

Formation damage is basically referred to as any unintended resistance to the fluid flow into or out of a wellbore. It restricts the flow by reducing permeability in the near wellbore region due to invasion of fluids used during drilling, completion, and workover operations (i.e. mud and etc.), into the reservoir rock. A low value of the permeability or high value of the skin factor would result in low well productivity.

A hydraulic fracture is a structure on a reservoir which remains undistributed out of the fracture. Having increased the contact surface between the well and the reservoir the well productivity is consequently improved. The hydraulic fracture improves the permeability of the damaged zone. The effective wellbore radius is defined to relate the productivity of well to the skin factor. It is defined as the radius 
that produces equivalent results to those obtained using a skin factor of zero. Acidizing method reduces the skin factor, therefor increases the effective wellbore radius.

\subsection{Transport in Heterogeneous media}

Homogeneity and heterogeneity are concepts relating to the uniformity in a substance. A material that is heterogeneous is non-uniform in composition or properties. Reservoirs are essentially heterogeneous assemblages of depositional rocks, each with different textures, types, bedding architectures, and characteristics. Heterogeneities can occur across all length scales within reservoir media.

According to their size or scale, geological heterogeneities have been classified in different categories as wellbore, inter-well, and field-wide scales of heterogeneity. Heterogeneities at the wellbore scale affect matrix permeability, distribution of residual oil, directional flow of fluids, potential fluid-rock interactions, and formation damage. Heterogeneities at the inter-well scale affect fluid flow patterns, drainage efficiency of the reservoir, and vertical and lateral sweep efficiency of secondary and tertiary recovery projects. Heterogeneities at the fieldwide scale determine the in-place hydrocarbon volume, areal distribution, and trends of hydrocarbon production. In a heterogeneous porous structure the macroscopic lumping of parameters is not sufficient to represent the heterogeneous systems where the flow is affected by local variations. 
The heterogeneities are more pronounced when dealing with subsurface processes, such as movement of ground water (Gelhar 1993; Gelhar and Axness 1983), transport of contaminants in groundwater, in-situ recovery of oil and natural gas (Miralles-Wilhelm, Gelhar and Kapoor 1997). The fact results from highly complex diagenesis processes of the sediments.

It is hard to get the adequate spatial and temporal data for mass and momentum parameters. It is mainly because heterogeneity always exists in the medium and no one knows how the variables change spatially. As a result, probabilistic framework gained the scientist attraction in the field of fluid flow in porous media. They characterize the pore structure with random quantities (for example the permeability, porosity, mineral concentration, internal specific surface area etc.). Permeability, in particular, is very important in fluid flow therefore is often used to describe the medium heterogeneity. Gelhar and Axeness studied the macroscopic dispersion coefficient through local spatial fluctuations in permeability (Gelhar and Axness 1983). They quantified the effect of the fluctuations on the transport and flow for a system that is statistically homogeneous. Others used their approach in different processes like contaminant plume degradation or variable density and viscosity fluids in aquifers.

This study similarly focuses on a wellbore scale of medium heterogeneity that affects the fluids behavior. It attempts to quantitatively describe the flow in porous media in a scale larger than an average pore size. In other words, the purpose is to find appropriate average parameters, which can be applied to flow, transport and reaction in the scale of interest. At the same time, being able to incorporate the 
influence of intrinsic porous medium heterogeneity on the modeling and predictions is often desired during an investigation.

\subsection{Introduction to Upscaling}

A geological model, to be used as an input for reservoir fluid flow simulation, contains millions of grid blocks. It would be ideal to integrate geological and petrophysical data at a scale that they are acquired. However, the restrictions in computing time, cost, and available resources make it nearly impossible without upscaling application. Upscaling is basically the process of clustering data into smaller sets of characteristics that represent the most significant aspects of a reservoir. Any upscaling will result in loss of data and cause error (Sablok and Aziz 2008). The models are based on the geological data that are usually in the fine scale. Then the fine scale models are upscaled to a coarse scaled model that will, to some extent, result in approximating geological complexities of the reservoir.

Reservoir simulation models are built by combining these coarse geological models with rock and fluid properties and other engineering data. Ideally, upscaling preserves the pressure profile from the fine scale model under the applicable flow process. The key steps in reservoir simulation are upscaling of the rock properties, primarily permeability, porosity and relative permeability, to reduce the number of grid blocks, and upscaling of the comprehensive compositional description of the reservoir fluid (Sablok and Aziz 2008). 
There are two main categories of upscaling methods: analytical, such as a wide range of averaging methods, streamlines, etc. and numerical, such as single and two phase flow simulations.

\subsection{Analytical Methods for upscaling}

The nonuniformity of the fluid flow and reaction controlling parameters in porous medium increase the fluctuations in velocity of the liquid front over a range of scales. Under the effect of these fluctuations, anomalous flow and transport happens, along with irregular deformation of the matrix itself. Various theoretical approaches have been developed in fields such as geological, biological and porous media to accurately predict transport and reaction process on a variety of natural scales. There are many techniques to investigate and upscale the effects of heterogeneous environments on fluid flow and reaction in porous media. Despite the difference among the various upscaling methods, the procedure of upscaling transport parameters include the three common operations: (1) characterization of the subsurface system at multiple scales; (2) estimation and evaluation of transport parameters at measurement scale; (3) upscaling transport parameters at a modeling scale.

In continuation, the most commonly used approaches are discussed.

\subsubsection{Integral Transform Methods}


Nonlocal theories, which are generally associated with evolving heterogeneity, are based on an integral transform or higher order gradient expansion. Evolving heterogeneity has two sources. One is the macroscale boundaries, which usually lead to nonlocal Darcy's law, and the other one is continuously evolving heterogeneity, such as encountered with fractal media.

One of the new powerful numerical methods used for general purposes is the finite integral transform technique. In this technique, which is mainly used to get the exact solution of linear problems, the nonlinear PDEs are transferred to a coupled nonlinear system of ODEs that could be solved by easier numerical methods such as: finite differences, finite elements, boundary elements, and spectral methods. The generalized integral transform method was able to solve several linear and non-linear models recently using truncated Eigen functions expansions.

\subsubsection{Moment Methods}

Method of moments is a statistical method to estimate the population of parameters. First the population moments (unknown) should be formulated by the parameters of interest. For instance, derive an equation that relates the expected values of powers of the random variable under consideration to the parameters of interest. Then a sample, from which the population moments are estimated, is drawn and replaced by the population moments in the equations. The equations are then solved for the parameters of interest to get estimates of those parameters. 
The stochastic moment equations for solute transport in natural porous media are so complicated and impossible to solve analytically because of the complexities in hydraulic conditions. The existence of finite hydraulic boundaries and the statistical nonstationarity of hydraulic conductivity that is caused from distinct geological layers, zones, or facies are all examples of these complexities. The analytical solutions for the spatial moments of the mean concentration are acquired in the traditional stochastic theories due to their assumptions that greatly simplify the calculations (e.g. Dagan, 1982, 1984; Gelhar and Axness, 1983). Zhang et al. (2000) applied a Lagrangian perturbation method to develop a theory for solute flux in nonstationary flow fields. The predicted results are the mean mass flux and variance about the mean. The analytical moment equations are then solved numerically through a finite difference method.

\subsubsection{Central limit or Martingale methods}

CLT/Martingale methods are probabilistically based approaches to upscaling. The periodicity employed here is deterministic, not stochastic. The outline is laid down by (Bhattacharya and Gupta 1990) and focuses on a conservative solute in a saturated porous medium. The diffusion equation is assumed to be valid at the microscale so that the diffusion coefficient is the effective pore scale diffusion coefficient because the microscale is between the pore and Darcy scales. CLT is to be valid at each scale so the approach is good. Deterministic spatial periodicity or quasi-periodicity are the basics used to prove the validation at each scale. 


\subsubsection{Mathematical homogenization}

The conception of finding equivalent descriptions for highly heterogeneous phenomena gained attention of the scientific community and since then there has been a wave of new techniques to obtain these equivalent description in various contexts. In the mechanics literature, these techniques are universally nomenclatured as "upscaling techniques". The method of asymptotic expansions of (Bensoussan, Lions and Papanicolaou 1978) and the method of moments of (Brenner 1980) and (Aris 1956) qualify has some of those upscaling techniques. In mathematics literature, these techniques are referred to as "Homogenization".

Homogenization is an asymptotic method for upscaling parameters of periodic media (Ene, 1990). The basic idea for homogenization is as follows. Consider a porous medium with microscopic structure characterized by period 1 and a macroscopic structure with the characteristic length of L. A spatial scale parameter can be defined as $\varepsilon=1 / \mathrm{L}$. Instead of working on one function $u$, a family of functions $\mathrm{u}^{\varepsilon}$ is considered to find the limit of $\mathrm{u}^{\varepsilon}$ when $\varepsilon \rightarrow 0$. The limit is considered as the result of upscaling procedure for homogenization, which consists of finding differential equations that the limit satisfies. The asymptotic process $\varepsilon \rightarrow 0$ stands for the transition from microscopic to macroscopic scale. Hence, the upscaling process of homogenization is completed by making the microscopic scale approximate to zero (Hornung, 1997).

The study of dispersion in porous medium saw some new directions in the 70's and 80's. Remarkable contributions being the method of asymptotic analysis applied in the case of no net macroscopic convection (Bensoussan, Lions and 
Papanicolaou 1978) and the method of moments (borrowed from (Aris 1956)) applied to the case of spatially periodic porous medium (Berner 1980). The crucial idea in the cited references being the consideration of convection-diffusion phenomena at the pore scale and averaging them over the entire porous medium, via asymptotic expansions in (Bensoussan, Lions and Papanicolaou 1978), and via the method of moments in (Brenner 1980). This viewpoint of starting with pore scale phenomena followed by an averaging technique had appeared in (S. Whitaker 1967) in the context of volume averaging techniques.

In case there are not any restrictions on the structure of the heterogeneities, i.e. they are not periodic; there are more general approaches than periodic averaging (homogenization): $\Gamma$-convergence; H-convergence; G-convergence (De Giorgi 1984). The notion of H-convergence was introduced by (Tartar 1977) (Murat 1977) to study a wide class of homogenization problems for possibly non-symmetric elliptic equations. In case of probabilistic and stochastic heterogeneities there have been proposed approaches to upscaling (Kozlov S. 1978), (Bensoussan, Lions and Papanicolaou 1978). The theory of periodic homogenization gets interesting as explicit expressions for the effective coefficients are obtained in terms of the microscale parameters.

\subsubsection{Mixture and hybrid mixture theory}

Hybrid mixture theory (HMT) involves using the mathematical filtering theorems to average the microscale field equations to obtain equations at higher scales. At macroscale, the constitutive equations are formulated by exploiting the entropy inequality in the sense of (Coleman and Noll 1963). During upscaling 
(averaging), some microscale information is lost. However, the effect of microscale transport mechanisms and thermomechanical processes on macroscale behavior is incorporated. One significant advantage of upscaling first and then formulating the constitutive theory is that the material coefficients in derived relations appear at the macroscale. The macroscale material coefficients are easier to measure via experiments or obtain from other experimental studies using conventional methods. Upscaled equations are solved in macro and mesoscale representative elementary volumes (REV), which saves the computational time significantly in comparison to time needed for solving the microscale equations.

HMT was initially used to explain the thermomechanical behavior of swelling and shrinking porous media like clays (Achanta, Cushman and Okos 1994)(Bennethum and Cushman 1996)(Hassanizadeh and Gray 1979).

\subsubsection{Renormalization group techniques}

Wilson first developed the renormalization group to study critical phenomena in phase transition (Wilson 1971). It is also called renormalization approach wherein the heterogeneity at a particular scale controls an overall behavior for a unit cell of a fractured porous. This scale is considered step 0 of the renormalization procedure. At step $n$ the effective properties are resulted from renormalization of a set of calculated properties. Then step-by-step transformation gives those properties to a probability distribution function of $f(n)$ for the property (for example, permeability). In this method the effective parameters are evaluated by recurrent applications of renormalization group transformation from the basic cell to the entire domain of 
interest. This method is used to upscale permeability in fractured porous media (King 1989) (Gavrilenko and Guéguen 1998). Quantitative analysis of the anomalous (nonFickian) diffusion laws arising when disorder is 'relevant' is best handled by renormalization group methods.

\subsubsection{Continuous time random walks}

Montroll and Weiss introduced this method as a generalization of physical diffusion process to effectively describe anomalous diffusion, i.e., the super- and subdiffusive cases (Montroll and Weiss 1965). Continuous-time random walk (CTRW) is a generalization of a stochastic jump process with arbitrary distributions of jump lengths and waiting times. It begins with a finite volume discretization of a fractured porous medium for small scale diffusion equation (Noetinger and Estebenet 2000). A particle is then released jumping from one grid block to the next one. The transition probability is proportional to the transmissivity. The particle movement path can be defined by its coordinates on the computational grid and by a random function of time $\tau(\mathrm{t})$, when starts its random walk in the domain. The correlation function of $\tau(\mathrm{t})$ can be calculated by Monte Carlo simulations. In the end an integration of the correlation function with respect to $t$ is taken and the upscaled mass transfer coefficient is found.

Berkowitz, et al applied this approach to the transport of substances in groundwater and were able to provide a good description of the anomalous transport behavior that occurs because of medium heterogeneities (Berkowitz, et al. 2006). NonFickian (or anomalous) transport of contaminants has been observed at field and 
laboratory scales in a wide variety of porous and fractured geological formations. Over many years a basic challenge to the hydrology community has been to develop a theoretical framework that quantitatively accounts for this widespread phenomenon. CTRW formulations have been demonstrated to provide general and effective means to quantify non-Fickian transport

\subsection{Stochastic-convective approaches}

The spectral integral approach has been applied in atmosphere turbulence for many decades (Lumley and Panofsky 1964). Gelhar and his colleagues introduced this method into hydrogeology at the end of 1970s and in the early 1980s (Bakr et al. 1978; Gelhar and Axeness 1983). Its basic idea is to decompose a stationary random variable into its ensemble mean and random fluctuation with a zero mean. Subsequently, using the relationship between power spectral density and the fluctuation to construct the correlation function of the random variable, upscaling process can be done within the Eulerian or Lagrangian framework (Rajaram 1997). The spectral integral approach is limited to upscaling parameters for unbounded porous media.

Media heterogeneity causes stochastic fluctuations in flow velocity and the Fick's law of diffusion fails to describe hydrodynamic dispersion at field scale. As a result, the conventional convection dispersion equation cannot describe the mass transport in a natural hydrogeologic system properly [Gelhar et al., 1979; Smith and Schwartz, 1980; Matheron and DeMarsily, 1980; Dagan and Bresler, 1979]. However, in a laboratory scale column tracer experiment the convection-dispersion equation is 
deterministic in representing the experiment [Hashimoto et al., 1964; Rose and Passioura, 1971; Fried and Combarnous, 1971; Fried, 1975; Gupta and Greenkorn, 1974; Cameron and Klute, 1977; Yule and Gardner, 1978].

Researchers have been trying to find a solution for this disparity in the applicability of deterministic and stochastic dispersion representations for different length scales. They used stochastic transport methods to analyze the transport of dissolved pollutants in saturated aquifers based on the flow velocity mean and spatial covariance function. (Neuman 1979) reviewed different mathematical approaches for these methods. (Gelhar et al. 1979) used spectral methods to relate dispersion to the variability of hydraulic conductivity in a stratified porous medium, and a couple of years later (Gelhar and Axness 1981) studied macrodispersion in heterogeneous aquifers. Using a first order approximation of a stochastic convection-dispersion equation, they found that dispersion exhibited non-Fickian behavior early in the displacement process and for large time asymptotically approached a Fickian diffusion process. The dispersivity associated with the Fickian process was related to the statistical properties of the medium. Those authors derived expressions that relate an effective macrodispersion coefficient to the covariance function of the random velocity. A similar stochastic-convective transport approach based on a general flow ensemble concept was developed independently by (Simmons 1981). This latter work suggested that dispersion observed at any length scale can be represented as stochastic-convective transport by applying a probability density function for the macroscopic velocity variation. (Dagan and Bresler 1979) specifically emphasized calculation of the velocity probability density function for a lognormal distribution of hydraulic conductivity and steady infiltration conditions, under the condition that 
velocity correlation between layers vanishes and no transverse diffusion occurs. (Smith and Schwartz 1980) directly investigated the macroscopic dispersion caused by spatially heterogeneous hydraulic conductivity. They defined an array of varying conductivity blocks with spatial correlations and simulated the solute particle transport in it. A range of transport behavior between Fickian and non-Fickian dispersion was produced in realizations of the random flow system.

Non-Fickian behavior is a consequence of long range correlation in the velocity, which is imposed by spatial structure of the porous medium. Whereas the fickian transport results when velocity correlations are relatively short-range compared with the solute travel distance and there is sufficient mixing between random velocities. We want to formulate dispersive transport in porous media as a stochastic-convective process applied in any scale and will not necessarily depend on a Fickian diffusion representation.

When the solute travel distance increases the measured dispersion coefficients is also increased. This is a well-known consequence of different scales of heterogeneity in porous media (Fried and Combarnous, 1971), ( Pickens et al., 1978), ( Pickens and Grisak, 1981), (Gelhar et al., 1979), (Smith and Schwartz, 1980), Matheron De Marsily, 1980). The field scale dispersivity is bigger than the lab scale. The lab scale dispersivity coefficient is not reliable for making transport predictions in the field. Lab measurements of dispersivity are typically performed on homogeneous media. It is shown that dispersivity measurement is more representative of both the particular medium and flow geometry. The term dispersion could be used to define any deviation of solute concentration from that determined by purely convective flow with a mean velocity. 


\section{Chapter 2}

\section{Matrix Acidizing}

\subsection{Introduction}

Acidizing is primarily used to either stimulate a well to improve flow or to remove damage that is caused by invasion of the fluids that are used in completion or workover to the formation. Basically two types of acid treatments exist based on the injection rates and pressures. First is Matrix acidizing which uses the injection rates resulting in pressures below fracture pressure and the second is Fracture acidizing. Fracture acidizing is also known as Acid Fracturing wherein the hydraulic fluid or acid is pumped at a pressure higher than the formation breakdown pressure to fracture the formation.

In carbonate and sandstone reservoirs, matrix acidizing is generally applied to reduce a large skin resulting from permeability damage during completion. There are various materials that cause plugging; the migrating particles in the porous medium or 
precipitates that are produced by chemical or physical changes in the reservoir initial state. Also the liquid or gas that changes the relative permeability of the formation rock can cause the plugging action. The correct remedial action can be taken once the damage and its origin have been characterized. The operations on the well like drilling, completion, and work-over are all potential source of damage. As a result, various types of damage can coexist. The primary use of matrix acidizing is to remove this plugging and increase the well productivity. A matrix treatment process could have close to $50 \%$ improvement in natural production if there is no damage in the rock.

In matrix acidizing the acids dissolve the sediments and mud solids within the pores that are decreasing the permeability of the rock. This process makes the pores of the reservoir bigger so the hydrocarbons can flow easier. There are some practical limits in volumes and types of acid and procedures to perform an effective acidizing to get an optimum removal of the formation damage around the wellbore. A 5-28\% HCL is usually used for matrix acidizing. The flow of aqueous HCL through the carbonate and sandstone does not have a uniform acid front. The flow and dissolution create some flow channels called wormholes. In sandstone acidizing the reaction between acid and the solid takes place relatively uniformly along the reaction front. This results from the fact that the reaction is slower and the reactant is not continuously consumed in the matrix behind the reaction front. However, in carbonate acidizing, the mechanism has a very selective pattern that makes some high permeable channels while others can remain untouched. As a result, this process has not been understood as for the fundamental processes controlling flow, reaction, and dissolution in the media. 
A thorough understanding of the fluid flow is required to predict the outcome of acidizing treatment; to determine the acid penetration distance, dissolution, and its profile distribution. A homogeneous porous medium is assumed in most current models to describe the matrix acidizing in sandstone. In some models the formation is divided into layers wherein the properties such as minerals, permeability, and etc. in each layer are constant. However, the prediction by these homogeneous models differs from the effects of injected acid in reality. Sandstones always have small heterogeneities in flow and minerals properties. Matrix acidizing in this case is assumed to be influenced by small-scale variations in the permeability field.

\subsection{Mathematical Model for Matrix Acidizing}

\subsubsection{Chemical Reaction Model}

There are many basic chemical factors that control the reaction mechanism at the molecular level. The interest point in many hydrological problems is the chemical reactions in aqueous solutions. The chemical and physical behavior of such solutions characterizes the aqueous solute concentration continuum.

Chemical reactions that usually happen in groundwater are dissolution of minerals, adsorption solute and microbial reactions. A set of equation describing a space domain in which there are chemical species and minerals as a function of time can mathematically define the reactive transport phenomena. Reactive transport 
modeling has become an essential tool for the researchers in subsurface hydrology (Steefel, DePaolo and Lichtner 2005).

Mass balance equations on acid, minerals, and overall mass balance, and Darcy's law, was used to develop a three-dimensional analytical model to describe the process. The assumptions for the flow and transport equations on the fine scale are as follows:

1-Flow is assumed to be only liquid on a solid phase.

2- Fluid and rock are both incompressible

3-Gravity has no effect

4-Sorptions are neglected

5-Local Coefficient of bulk dispersion $\mathrm{D}_{\mathrm{ij}}$ is constant

6-'small' random perturbation distribution about the mean occur in liquid velocity, tracer and mineral concentrations, and reaction rate $\omega$.

In this study the fluid flows and transports the solute in porous media while there is an interaction between solute and solid. A first-order heterogeneous reaction takes place between the injected liquid solution and a stationary solid residing on the grain surfaces of the inert porous matrix. The system is an isothermal reactive flow system. The overall reaction is considered to be:

$$
[\text { Mineral }]+\tilde{v}[\text { Tracer }] \rightarrow \tilde{v}_{p}[\text { Product }]_{\mathrm{aq}} .
$$


Since the process involves a heterogeneous reaction, a tracer-mineral reaction wave develops spontaneously and propagates at a velocity less than that of the injected fluid (Durlofsky, Jones and Milliken 1997). It is within this moving boundary, where the mineral dissolution occurs at relatively high rates. Outside the reaction wave, the problem is mainly controlled by convective mass transport, initial and boundary conditions. The porous medium is described with fixed permeability heterogeneity.

\subsubsection{Governing Equations for Acid Solution}

The governing equations, mass balance of the tracer, for a planar reaction wave propagating in a homogeneous porous medium is:

$\phi \frac{\partial}{\partial t}(Y \rho)+\frac{\partial}{\partial x_{i}}\left(Y \rho v_{i}\right)=\frac{\partial}{\partial x_{i}}\left(\phi D_{i j} \rho \frac{\partial Y}{\partial x_{j}}\right)-(1-\phi) v \rho_{m}^{o} W$.

Where $\mathrm{Y}$ is the tracer concentration, $D_{i j}$ is the tracer dispersion coefficient tensor in liquid phase which includes all dispersion effects due to fluctuations on microscopic scales, $v_{i}$ linear velocity, $v$ represents the mass-weighted stoichiometric coefficient for the tracer defined in terms of the stoichiometric coefficient $\tilde{v}$ as follows 
$v=\frac{\tilde{v} M_{t}}{M_{m}}$

Here, $M_{t}$ and $M_{m}$ are molecular weights of the tracer and mineral respectively.

Finally, the local flow is represented by

$v_{i}=-\left(\frac{K_{i j}}{\mu}\right) \frac{\partial p}{\partial x_{i}}$

Where $K_{i j}$ is the absolute permeability and $\mu$ is the concentration-independent liquid viscosity. Total liquid mass balance is

$\phi \frac{\partial \rho}{\partial t}+\frac{\partial}{\partial x_{i}}\left(\rho v_{i}\right)=0$

$\rho_{m}$ is the dissolvable mineral mass per total volume and describes the quantity of reactive mineral $\rho$ is the density of the carrier liquid, independent of tracer and aqueous product concentrations. Because the acid is usually consumed during the reaction, the rate of the appearance of acid in the solution is negative. The mineral mass balance is:

$\frac{\partial \eta}{\partial t}=-W$

Here the depth of mineral conversion is represented by $\eta=\rho_{m} / \rho_{m}^{o}$, and $W$ the mineral dissolution rate. Hence, $\eta$ corresponds to a normalized solid mass that is 
available for reaction at a particular time and location. Using the law of mass action, the dissolution rate will be taken as follows:

$W=k_{0} a_{s}^{n_{1}} Y^{n_{2}} \eta^{n_{3}}$,

Reaction rate is basically defined as the appearance rate of species in the solution in a particular time period. In the equation above, exponents $n_{i}$ represent the dependences on mineral surface area $a_{s}$ and the concentrations of the reactants and $k_{0}$ is the coefficient of reaction.

The reaction rate expression is further simplified by taking the exponents $n_{i}=1$, and generating a new reaction rate coefficient:

$\omega=k_{0} a_{s}$

Hence, the reaction rate is now given as $W \approx \widetilde{r}=\omega Y \eta$. In addition, we introduce a dimensionless parameter $\alpha=\left(v \rho_{m}^{o}\right) /\left(\rho_{0}\right)$ representing the tracer reactant capacity. The new governing equations for the reactive flow would be:

$\phi \frac{\partial Y}{\partial t}+\frac{\partial}{\partial x_{i}}\left(v_{i} Y\right)=\frac{\partial}{\partial x_{i}}\left(\phi D_{i j} \frac{\partial Y}{\partial x_{i}}\right)-(1-\phi) \alpha \tilde{r}$,

$\frac{\partial \eta}{\partial t}=-\tilde{r}$

$$
\frac{\partial v_{i}}{\partial x_{i}}=0
$$


The first term on the left hand side of the equation $8 \mathrm{a}$ is a transient term that represents mass of acid by its concentration in the liquid, Y. The second term on the left side represents the transport of acid by convection, defined as Darcy flow. The first term on the right side represents the transport of acid by dispersion, defined as Fickian diffusion. Some previous studies neglected the effect of dispersion compared to the spread of acid front by chemical reactions. For mass balance of acid in this work the reaction is the source term, the second term on the right side of equation.

Two main parameters affect the overall rate of acid consumption or mineral dissolution. One is reaction rate and the second is the rate of transport of acid to the mineral surface.

\subsubsection{Species behavior in the Matrix-Homogeneous Case}

The homogeneous governing equations are numerically solved using finite difference method. For this matter, the terms of the equations are first turned into nondimension ones by changing the characteristic parameters to the following terms,
$Y^{*}=\frac{Y}{Y_{0}}$
$\eta^{*}=\frac{\eta}{\eta_{0}}$
$x^{*}=\frac{x}{x_{0}}$
$\tau=\frac{t D}{L^{2}}$

The governing flow equations of the injecting fluid are read in a dimensionless in the forms below:

$\frac{\partial Y^{*}}{\partial \tau}+\frac{L v}{D_{i j} \phi} \frac{\partial}{\partial x^{*}{ }_{i}}\left(Y^{*}\right)=\frac{\partial}{\partial x^{*}{ }_{i}}\left(\frac{\partial Y^{*}}{\partial x^{*}{ }_{i}}\right)-\frac{L^{2} \eta_{0}}{D_{i j} \phi}(1-\phi) \alpha \omega Y^{*} \eta^{*}$ 
$\frac{\partial \eta^{*}}{\partial \tau}=-\frac{L^{2} Y_{0}}{D_{i j}} \tilde{r}$

Initially the concentration of acid in the media is zero, $Y_{0}^{*}=0$, and the entire reactive mineral (which is $10 \%$ of the volume of the formation rock, $\eta_{0}=0.1 \mathrm{~mol} / \mathrm{cc}$ solid) is available for the reaction with acid, $\eta_{0}^{*}=1$. Assuming no acid flows out of the medium, Neumann zero boundary conditions $\frac{\partial Y_{x=L}^{*}}{\partial \tau}=0$ is selected for $\mathrm{y}$ at infinity (in the flow outlet where $x^{*}=1$ ). The acid entering the media has a constant concentration of $0.05(\mathrm{~mol} / \mathrm{cc}$ pore $)$ in the inlet. As a result, we choose a Dirichlet zero boundary condition for acid in the inlet $Y_{x=0}^{*}=1$. Constant pressure, or no flow boundary, is the most straightforward boundary condition. As far as the mineral distribution concerns, assuming the stationary mineral in the pore space, we choose Neumann zero boundary condition for $\eta$ at the point of the flow entrance and exit, $\frac{\partial \eta_{x=0}^{*}}{\partial \tau}=0$, respectively. Although the coarse properties obtained in the results may be associated to certain levels of ambiguities, this boundary condition is widely used in practice because of its analogy to one-dimensional core flood experiments (Warren and Price 1961)where the pressure drop is imposed between the inlet and outlet of a core to determine macroscopic properties such as porosity and permeability.

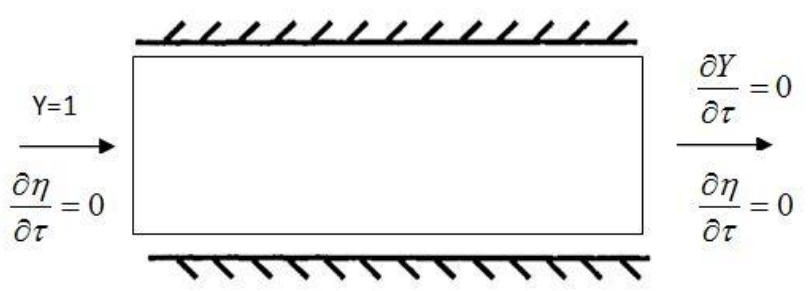

Figure 1. Schematic of constant pressure no flow boundary condition 
In continuation, using the values given in Table. 1 a numerical analysis is performed and the acid and mineral concentration for different times and locations illustrated.

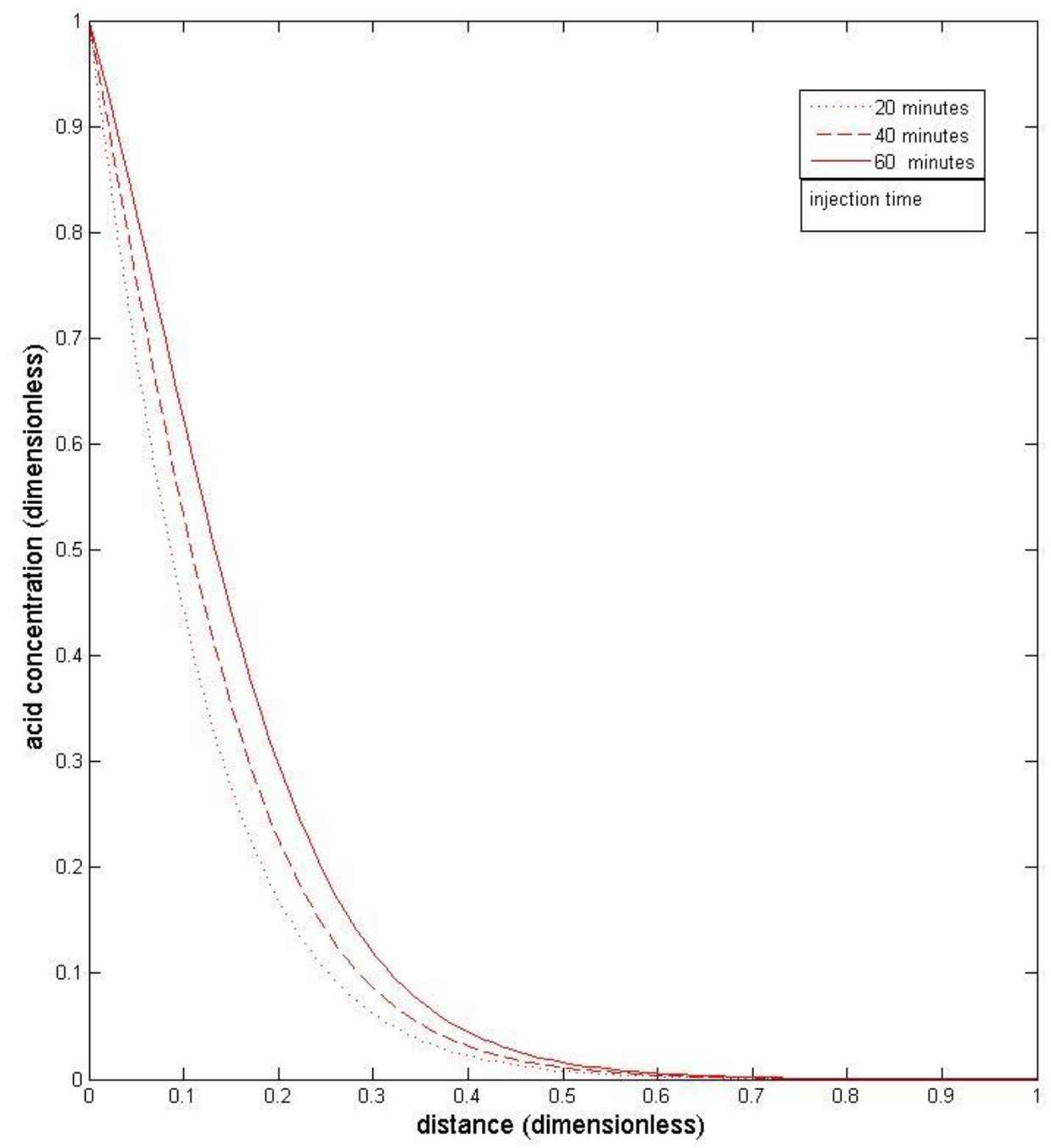

Figure 2. Early time effect of injection times on Acid concentration profiles 


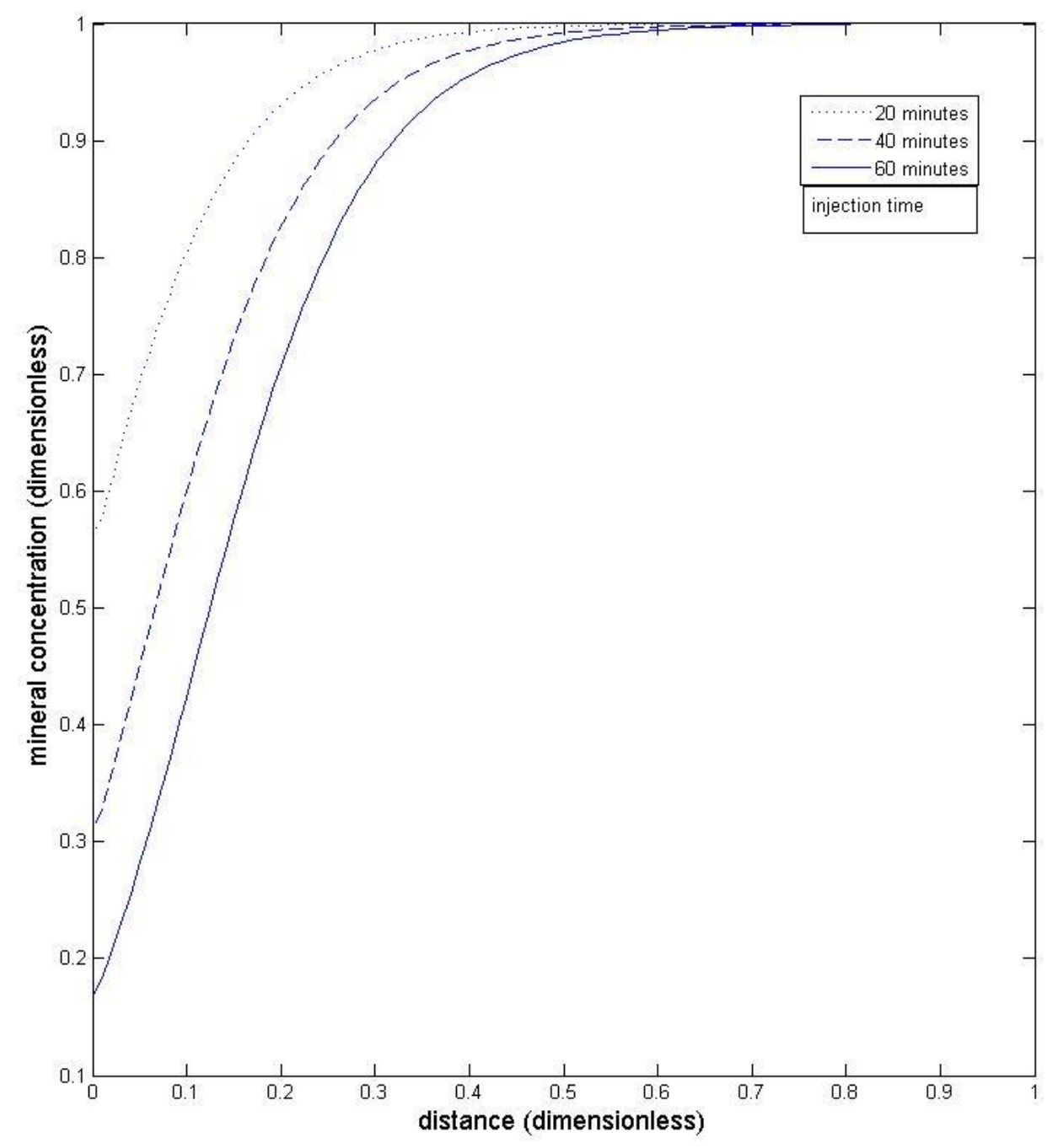

Figure 3. Early time effect of injection times on Mineral concentration profiles

In figure 1 the acid concentration profile is going toward higher values as the injection time has passed. Figure 2 approves the fact that as the injection time increases more mineral is consumed and the mineral concentration distribution tends to approach towards lower values and eventually at time infinity all the mineral is reacted and washed away from the medium. The figure below is shown to describe the process for longer injection times. 
As figure 5 suggests, the recovery of the minerals in the outlet of the medium reaches above $95 \%$. It is also found in figure 6 that due to non-existence of mineral in the medium the acid concentration barely drops from its initial value.

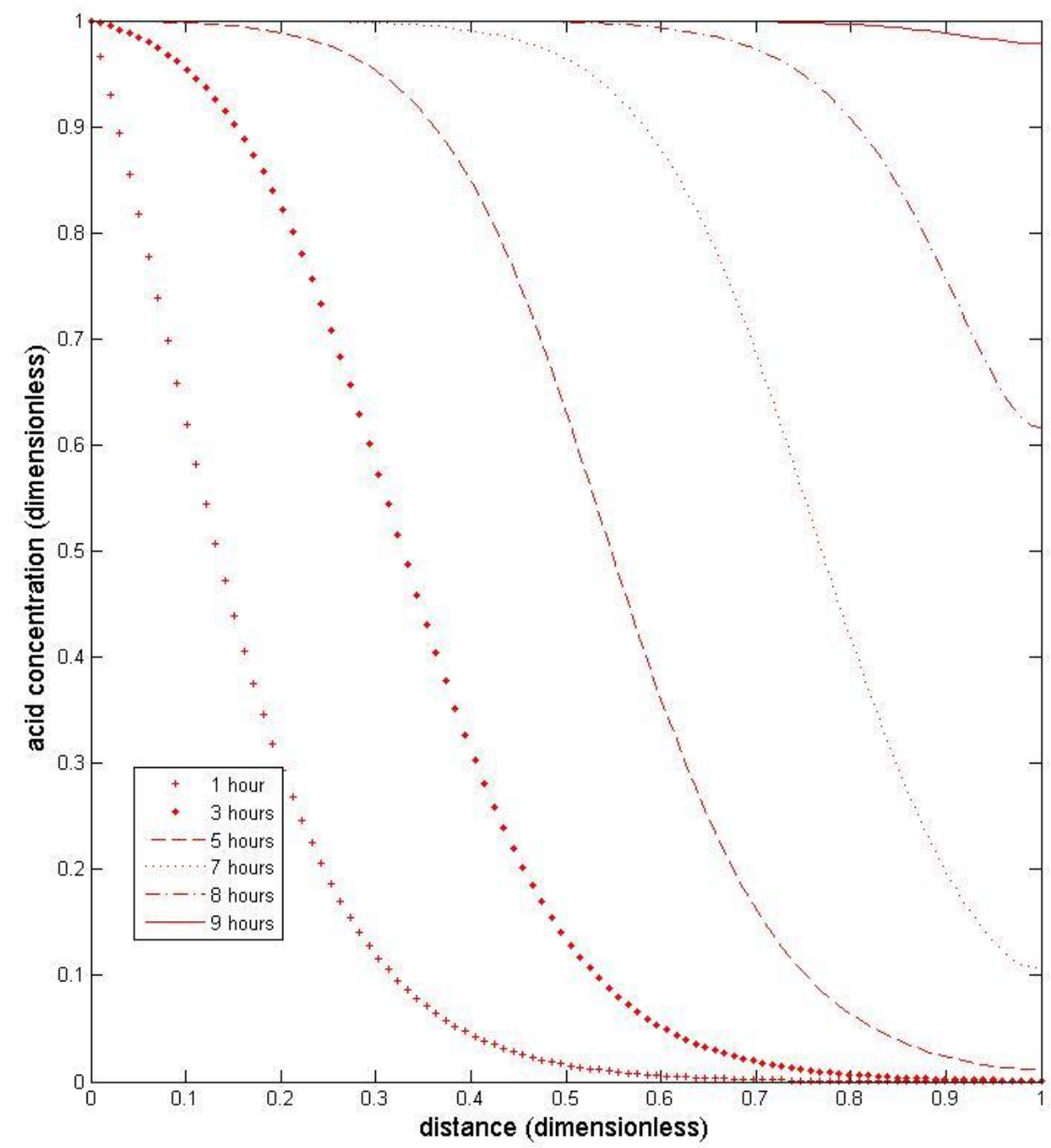

Figure 4. Demonstration of acid concentration distribution in long injection times 


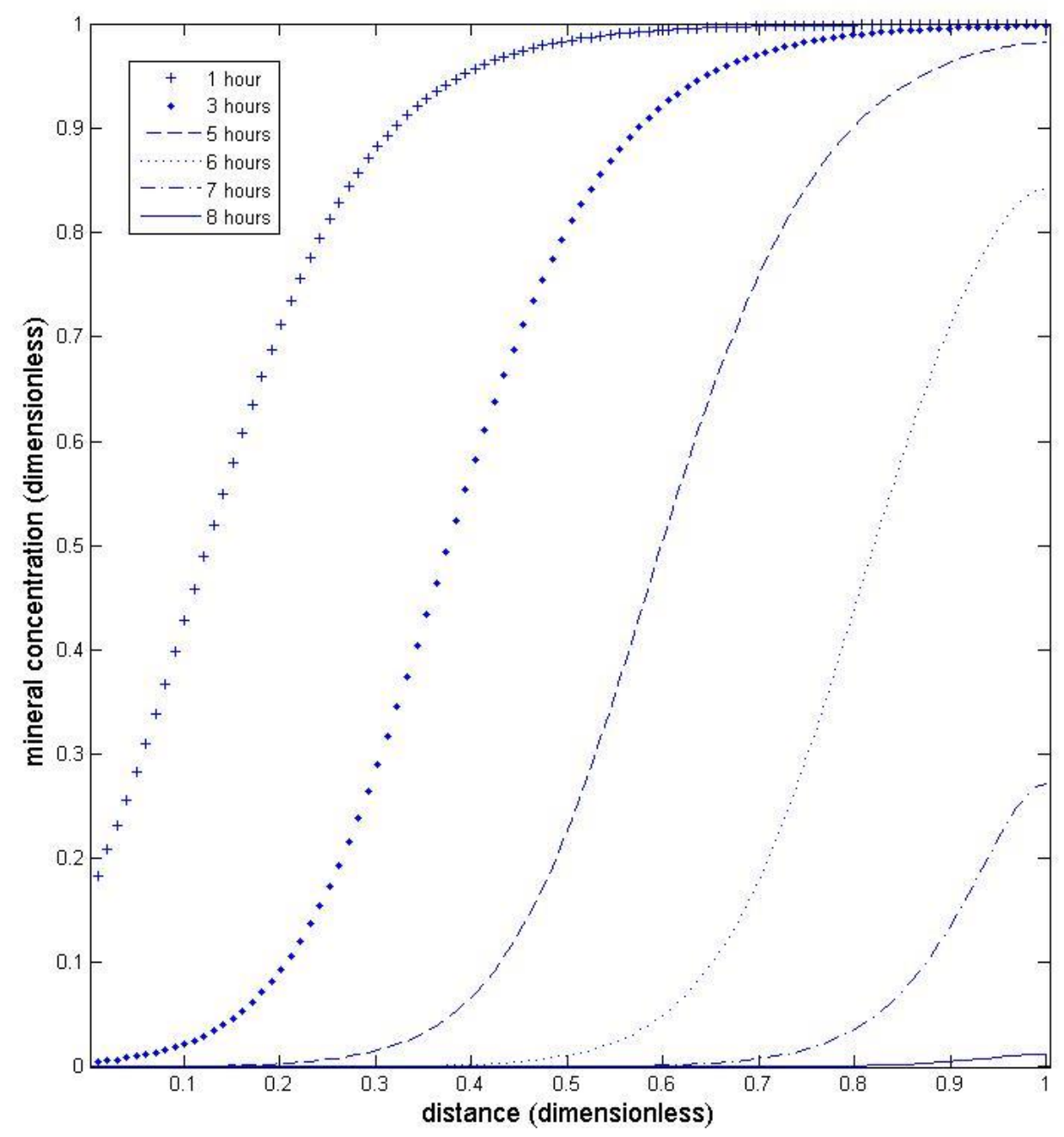

Figure 5. acidizing effect on the mineral concentration in long times 


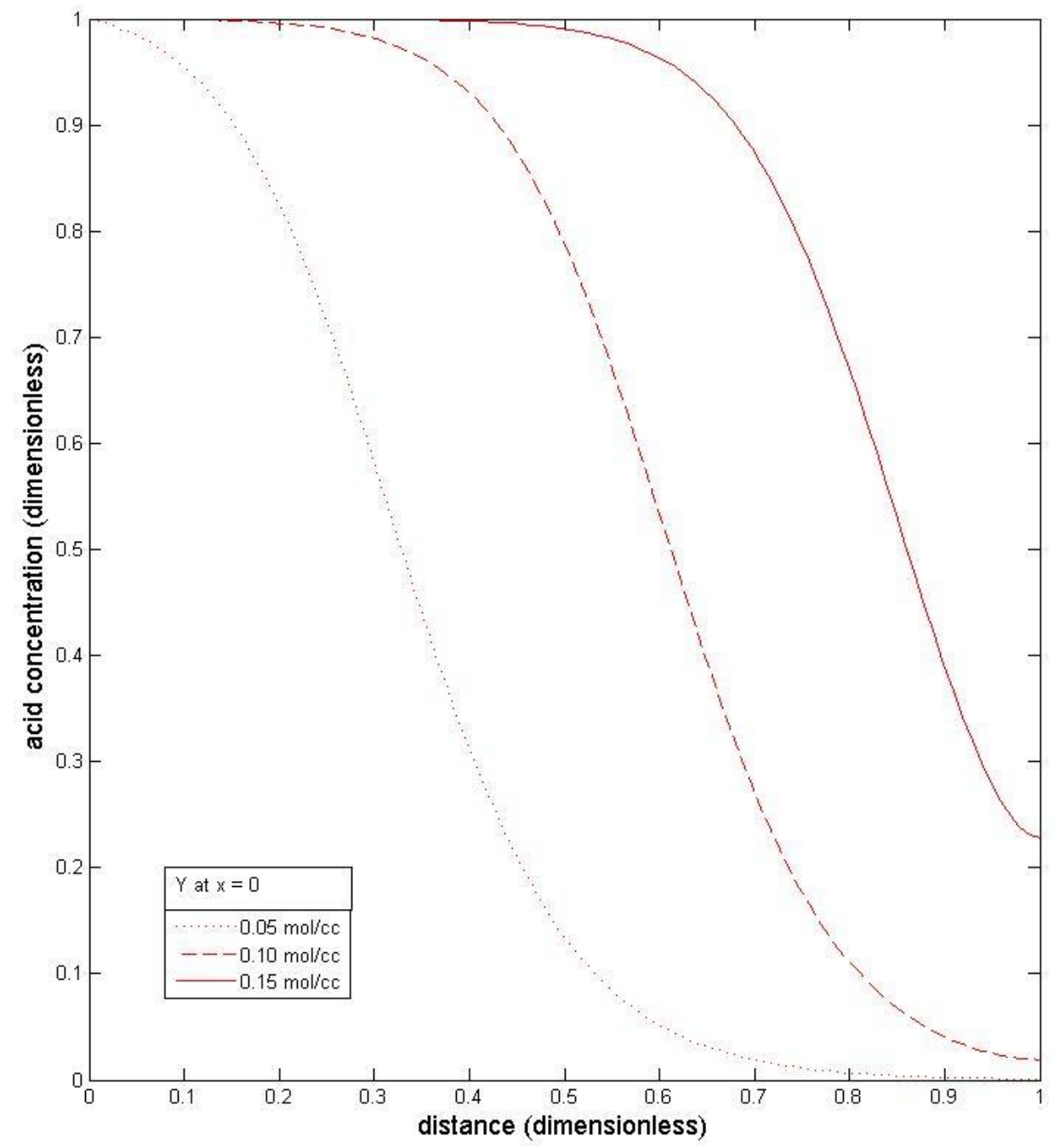

Figure 6. Effect of injection fluid acid concentration on Acid profile after $3 \mathrm{~h}$ 


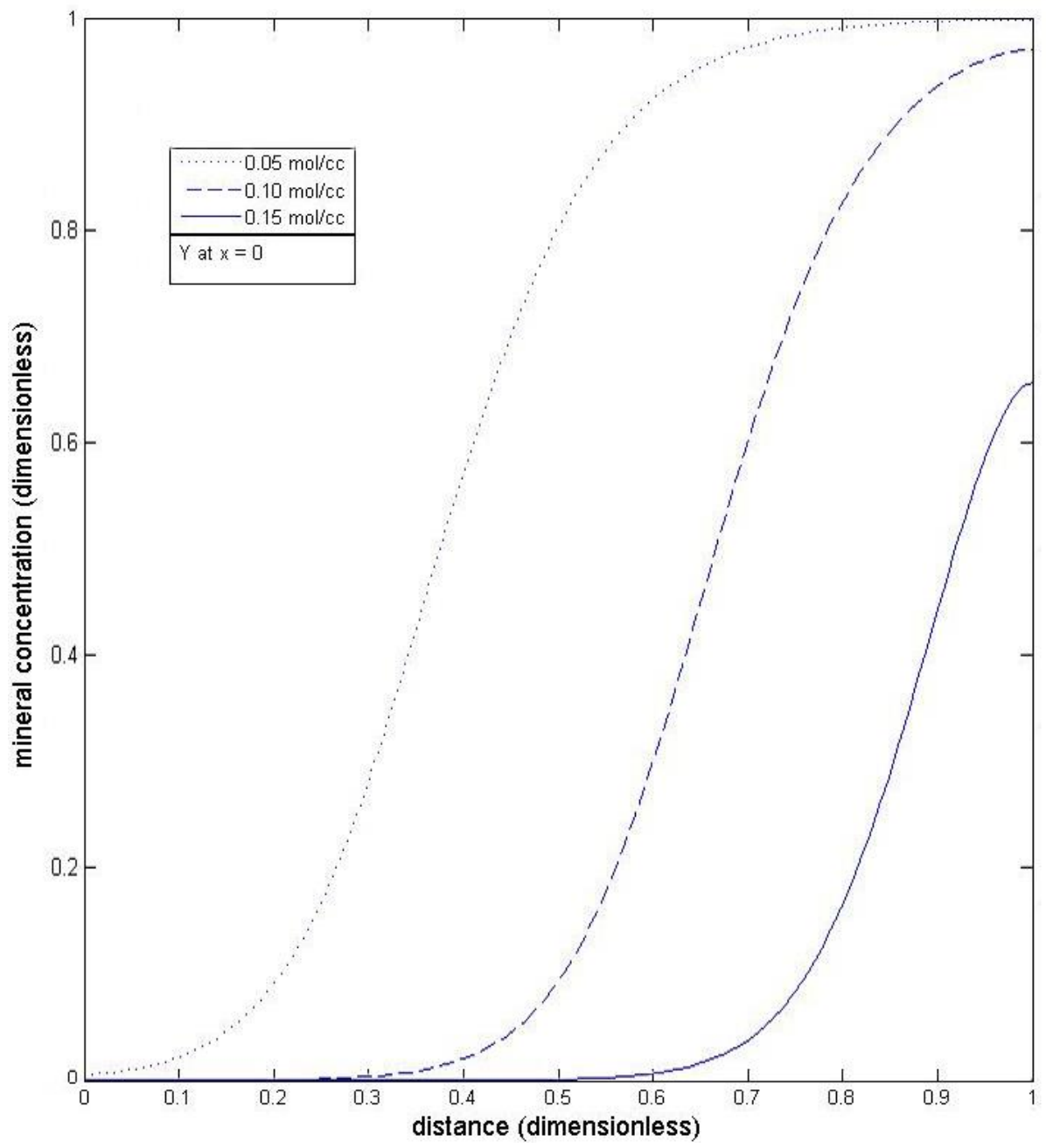

Figure 7. Effect of injection fluid acid concentration on mineral profile after $3 \mathrm{~h}$

In figure 5 and figure 6 one could interpret that the higher acid concentration of the injecting fluid the more mineral consumed. This arises from the fact that in chemical reactions higher concentration of the reactants provides a higher rate to the reaction. 


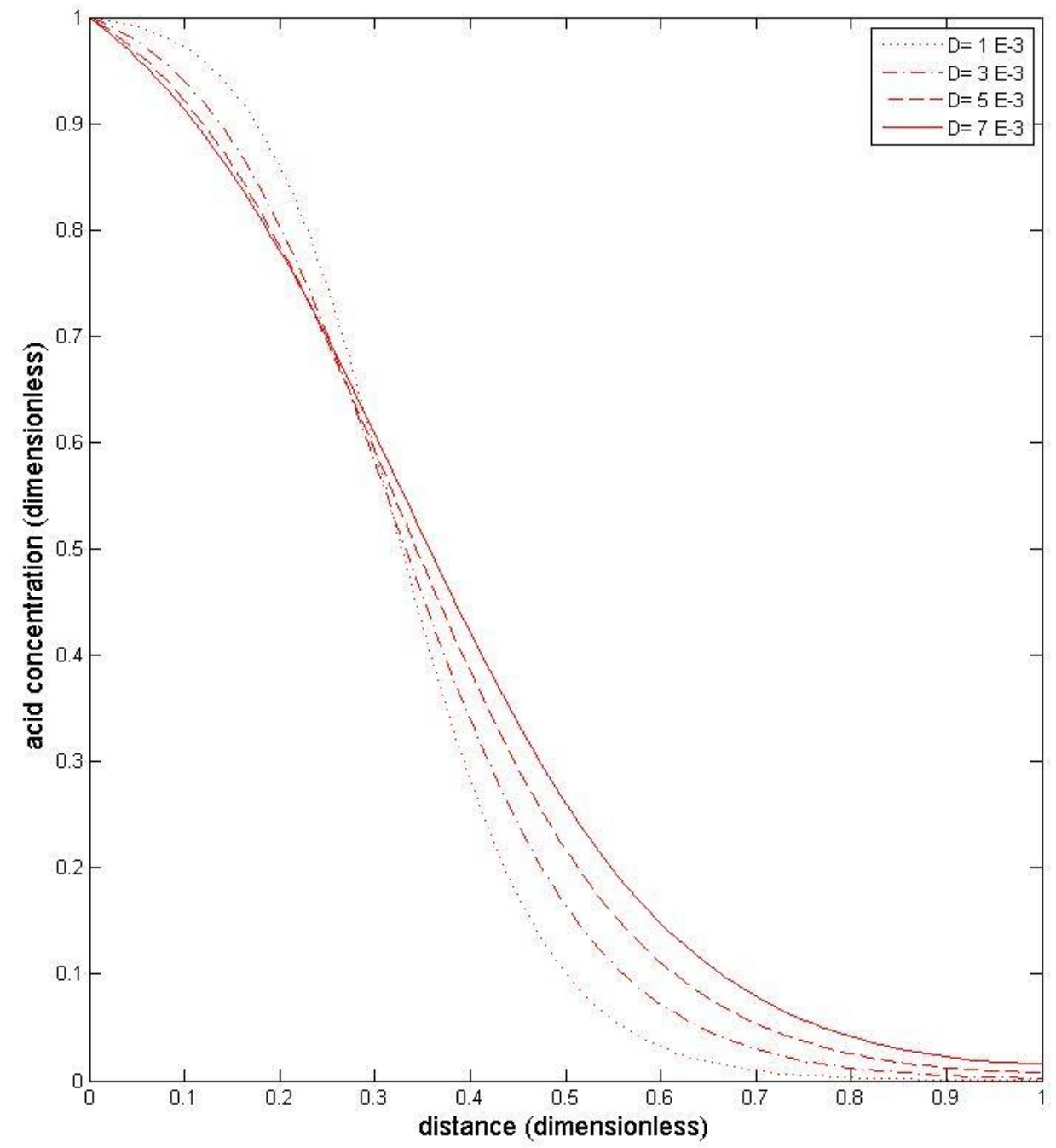

Figure 8. Effect of diffusivity on acid distribution 


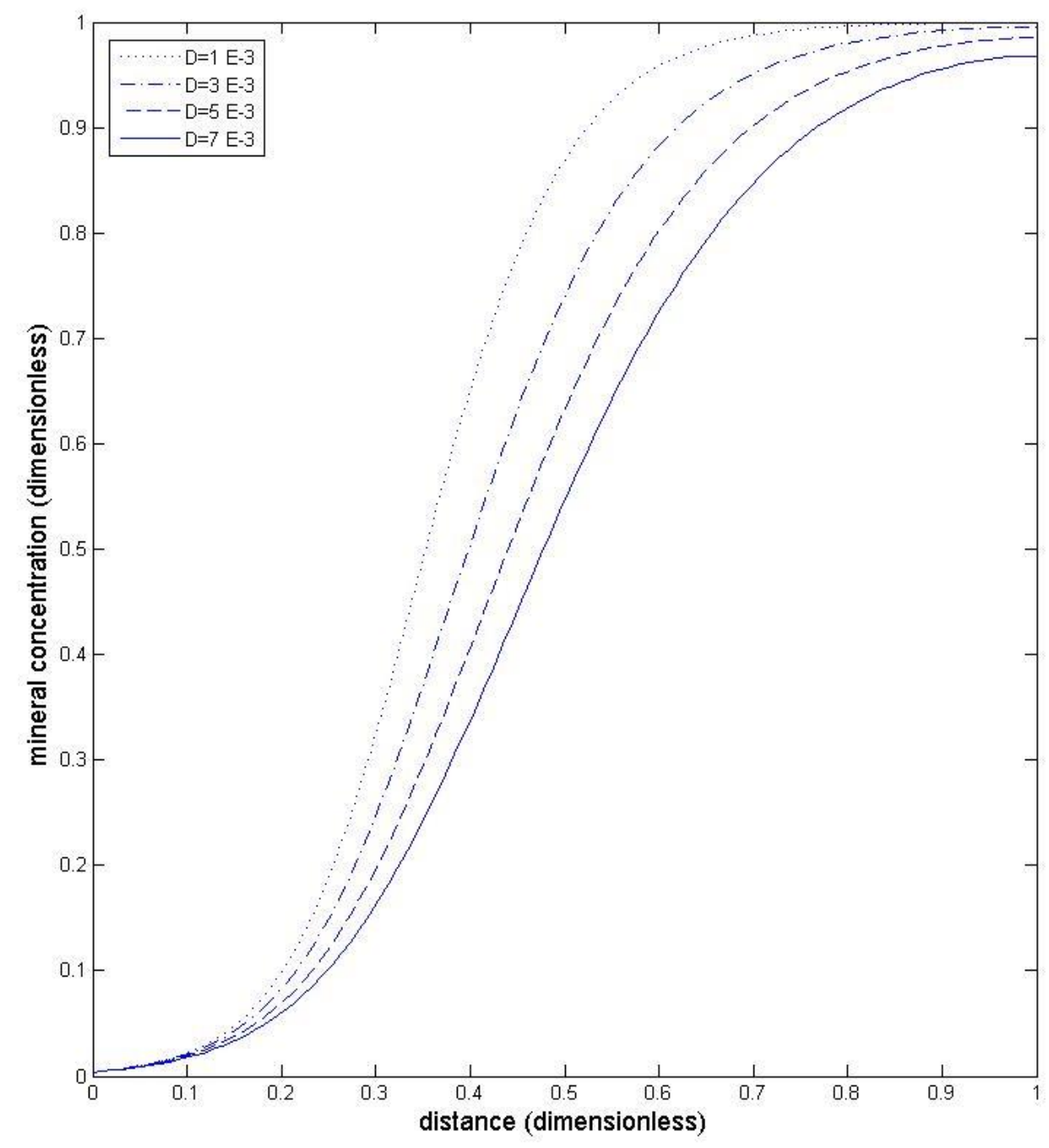

Figure 9. Effect of diffusivity on mineral distribution

Figures 9 shows that the higher the diffusivity coefficient the more fraction of the mineral reacted, and accordingly the less amount of acid involved in the reaction. This difference is pronounced where the process is far from the boundaries. In figure 8 the effects of the diffusion will apply after the fluid is past the convective-driven concentration region. 


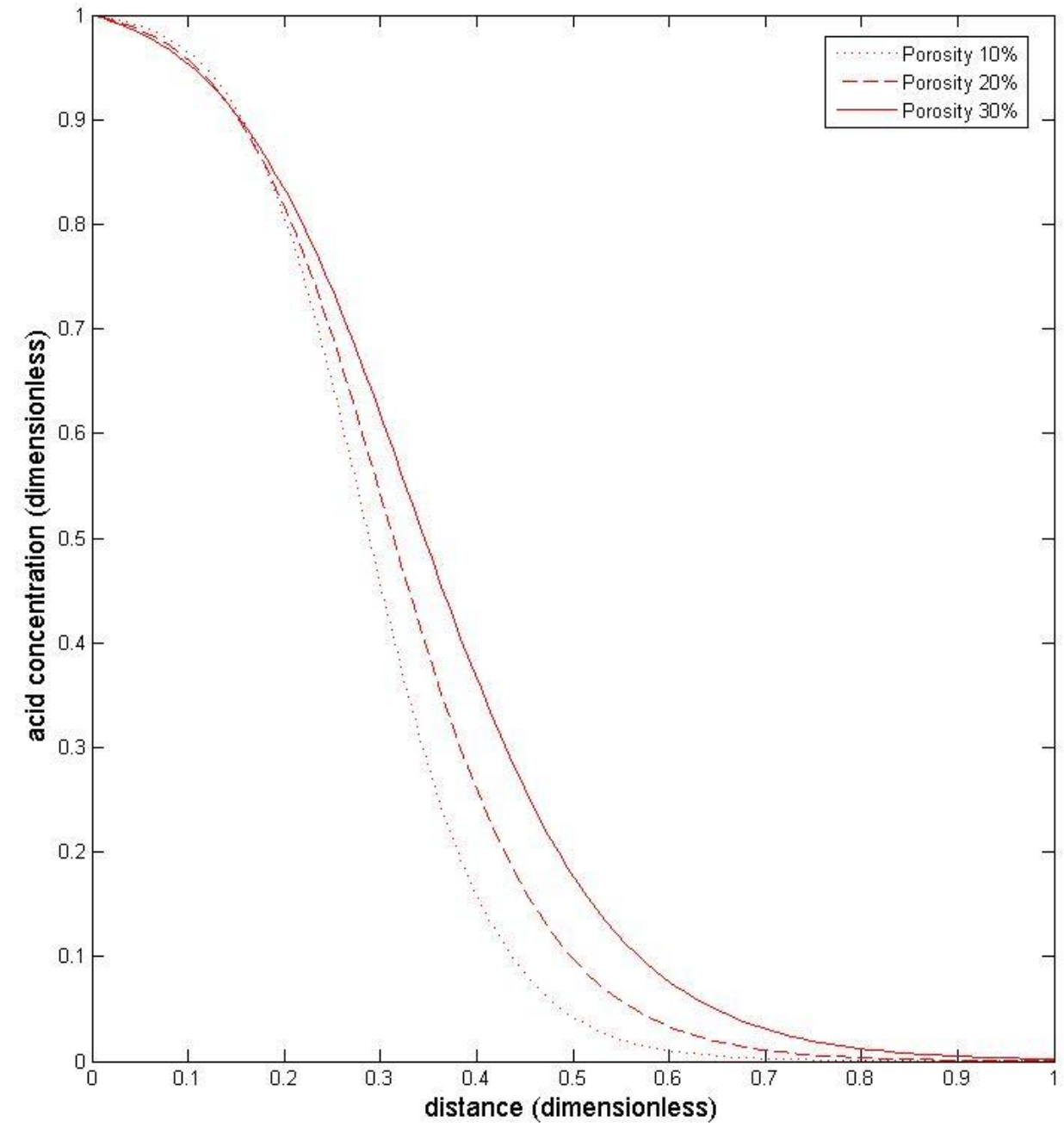

Figure 10 . Impact of porosity on acid distribution 


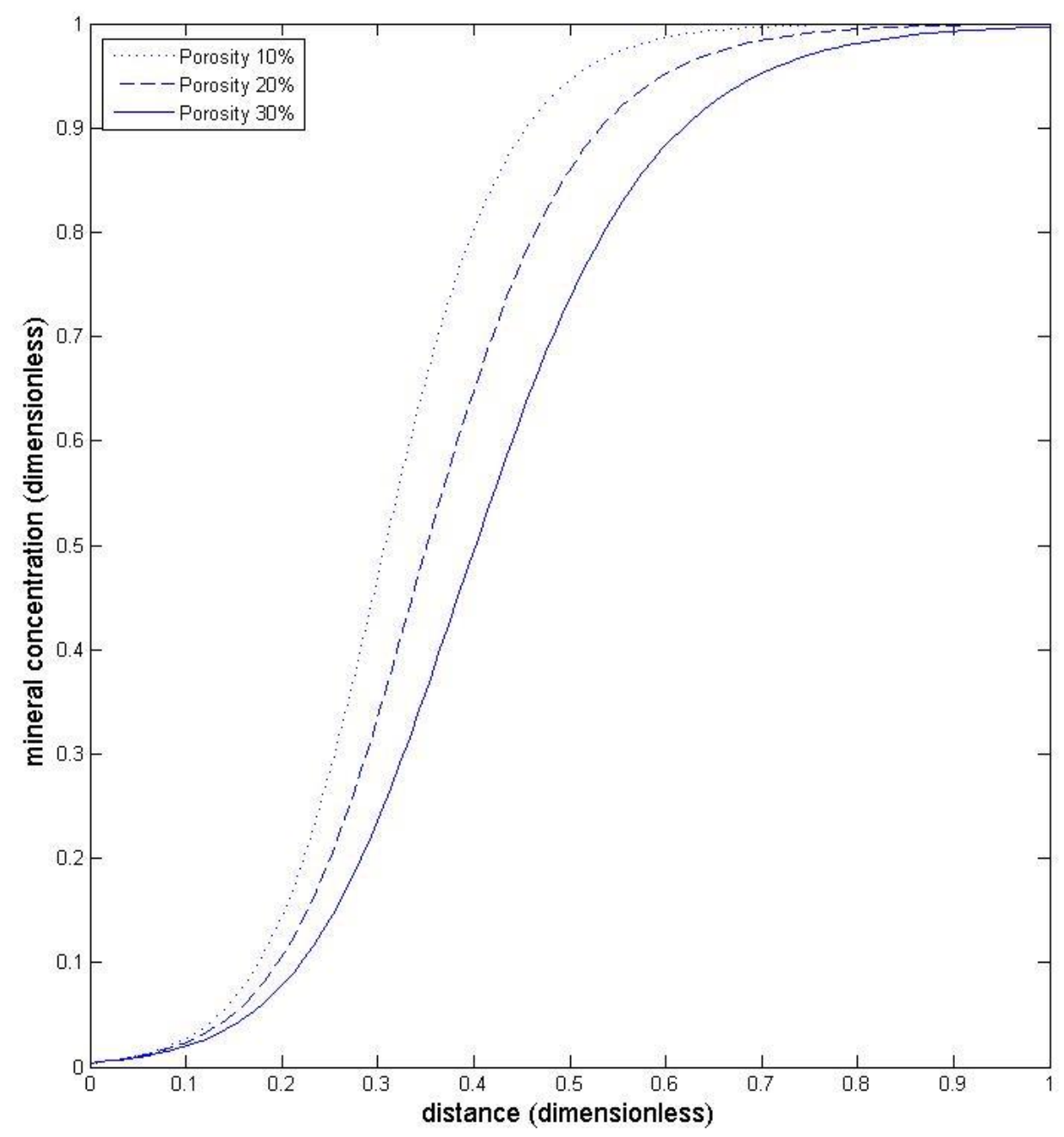

Figure 11. Impact of porosity on mineral distribution

Figure 10 and 11 also show that higher porosities render the process of acidizing to some extent. Having higher amount of surface area, that is bigger porosity values, results in faster reaction. The two figures confirm the latter physical behavior. 


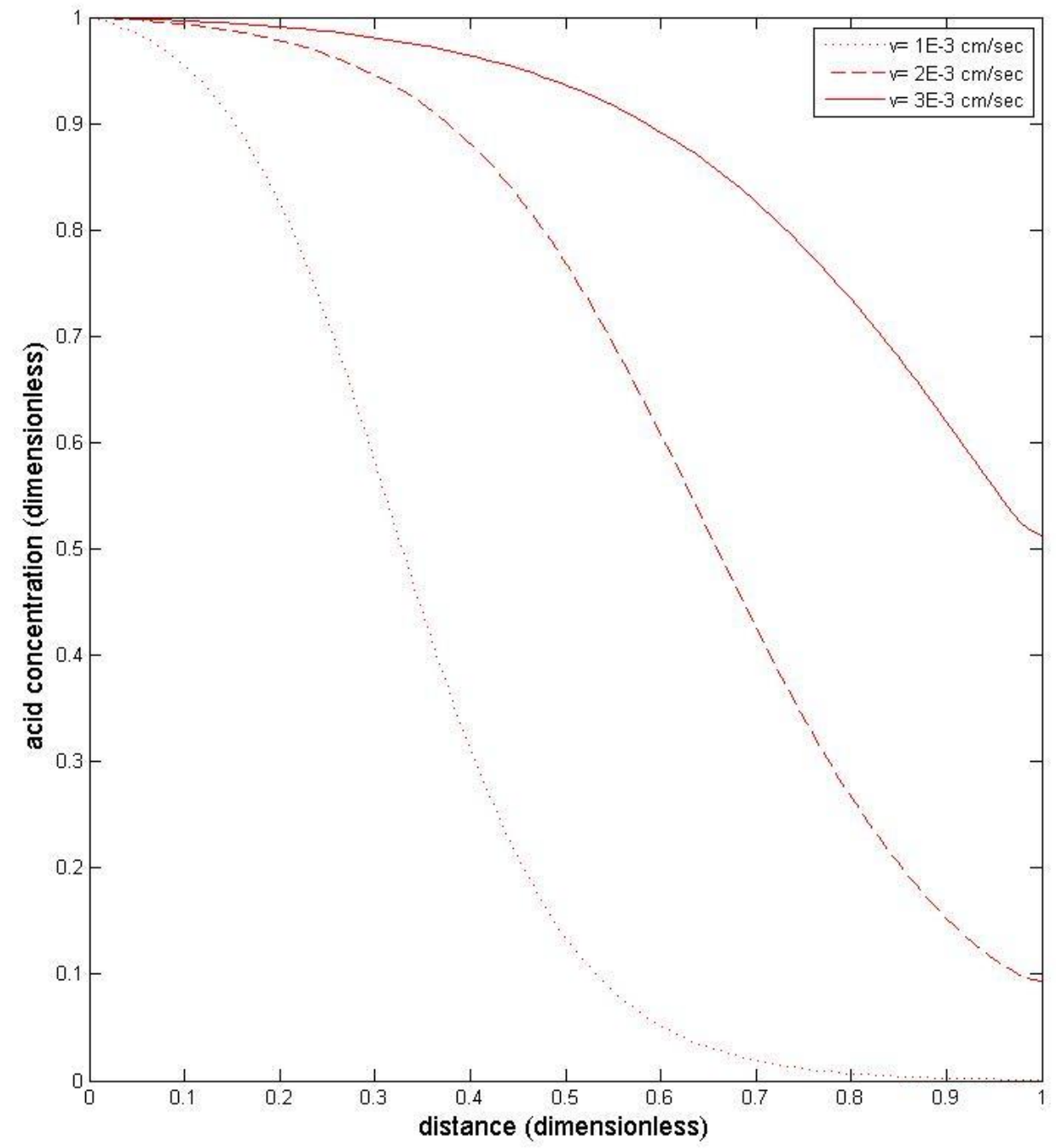

Figure 12. Acid distribution and different injecting acid linear velocities 


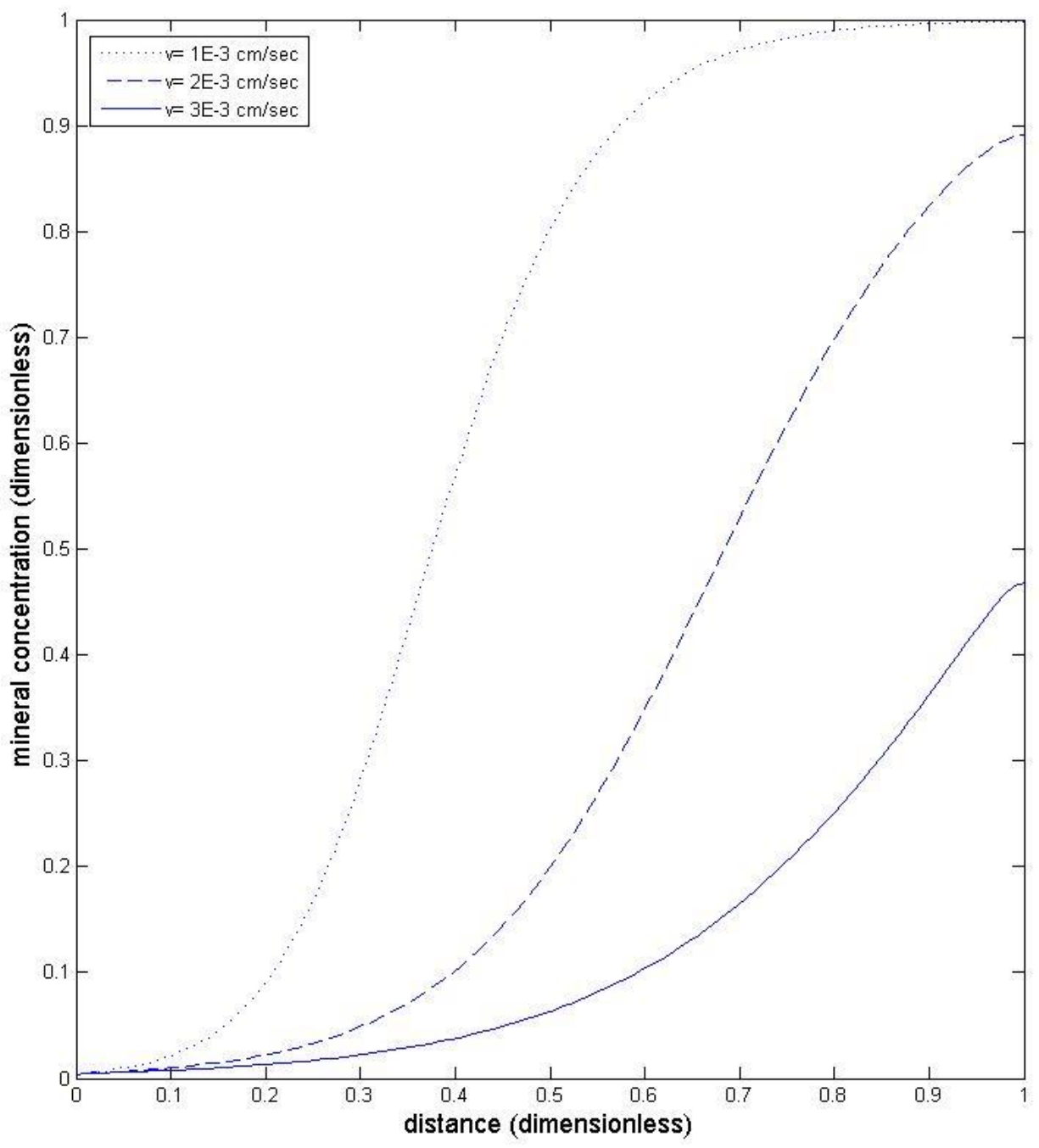

Figure 13. Mineral distribution and different injecting acid linear velocities 
Table 1. Parameters of Advection-Reaction Problem in Homogeneous Porous Media

\begin{tabular}{|c|l|}
\hline Parameter & Value \\
\hline$\varnothing$ & 0.25 \\
\hline$v\left(\mathrm{~cm} / \mathrm{sec}^{\prime}\right)$ & 0.001 \\
\hline$\alpha$ & 2.0 \\
\hline $\bar{\omega}$ & 0.01 \\
\hline$D_{i j}\left(\mathrm{~cm}^{2} / \mathrm{sec}\right)$ & 0.002 \\
\hline$L_{(\mathrm{cm})}$ & 10 \\
\hline $\left.\mathrm{Time}_{\mathrm{sec}}\right)$ & 10800 \\
\hline
\end{tabular}




\section{Chapter 3}

\section{Methodology}

\subsection{Random Fields}

The heterogeneity is introduced to the porous medium through a time-independent, spatially variable random permeability field. This field is defined with a mean value $\bar{f}$ and its small perturbation $f^{\prime}$ :

$f\left(x_{i}\right)=\bar{f}+f^{\prime} ; \quad E[f]=\bar{f} ; \quad E\left[f^{\prime}\right]=0$.

The spatial variations are quantified by a three dimensional statistically homogeneous anisotropic exponential covariance with the following spectral density function:

$S_{f f}=\frac{\sigma_{f}^{2} \lambda_{1} \lambda_{2} \lambda_{3}}{\pi^{2}\left[1+\left(k_{1} \lambda_{1}\right)^{2}+\left(k_{2} \lambda_{2}\right)^{2}+\left(k_{3} \lambda_{3}\right)^{2}\right]^{2}}$

Where $\lambda_{i}$ and $k_{i}$ are components of correlation length and wave number, respectively (Gelhar and Axness 1983). During the flow, the liquid velocity, tracer and mineral concentrations and $\omega$ are influenced by the medium heterogeneity and hence are represented in terms of their means and perturbations accordingly: 


$$
\begin{array}{lll}
Y=\bar{Y}+Y^{\prime} ; & E[Y]=\bar{Y} ; & E\left[Y^{\prime}\right]=0 ; \\
\eta=\bar{\eta}+\eta^{\prime} ; & E[\eta]=\bar{\eta} ; & E\left[\eta^{\prime}\right]=0 ; \\
v_{i}=\bar{v}_{i}+v_{i}^{\prime} ; & E\left[v_{i}\right]=\overline{v_{i}} ; & E\left[v_{i}^{\prime}\right]=0 ; \\
\omega=\bar{\omega}+\omega^{\prime} ; & E[\omega]=\bar{\omega} ; & E\left[\omega^{\prime}\right]=0 ;
\end{array}
$$

A partial linear correlation with intercept $a$ and slope $b$ is assumed for the reaction rate coefficient $\omega$ with $f\left(x_{i}\right)$, or permeability, in space as followed:

$$
\omega=a+b\left(f-f_{\min }\right)+\delta ; \quad \quad \bar{\omega}=a+b\left(\bar{f}-f_{\min }\right) ; \quad \omega^{\prime}=b f^{\prime}+\delta .
$$

$a$ is equal to $\omega_{\max }$,corresponding to its minimum counterpart in the permeability field, when a negative correlation is selected $(b<0)$. The correlation residual $\delta$ is assumed to be statistically homogeneous random field described by a three-dimensional anisotropic exponential auto-covariance function, with a spectrum given in Appendix B.

\subsection{Spectral Analysis}

The coordinate system $x_{i}$ is chosen such that $x_{1}$ is the direction of the mean flow, hence, we take:

$$
\overline{v_{1}}=v ; \quad \overline{v_{2}}=\overline{v_{3}}=0 .
$$


The perturbations in velocity $v_{2}^{\prime}$ and $v_{3}^{\prime}$ are not zero. Approximately the local dispersion tensor is

$$
D_{i j}=\alpha_{T} v \delta_{i j}+\left(\alpha_{L}-\alpha_{T}\right) \frac{\overline{v_{i}} \overline{v_{j}}}{v}
$$

$\alpha_{L}$ and $\alpha_{T}$ are the local longitudinal and transverse dispersivity values and $\delta_{i j}$ the Kronecker delta function. Then for the reaction term we use Taylor series expansion centered on the mean concentrations is used as a function of the mean and perturbed quantities:

$\tilde{r}(\omega, Y, \eta) \approx \omega\left(r+r_{Y} Y^{\prime}+r_{\eta} \eta^{\prime}+r_{Y Y} Y^{\prime 2}+r_{\eta \eta} \eta^{\prime 2}+r_{Y \eta} Y^{\prime} \eta^{\prime}\right)$

with

$r=\frac{1}{\omega} \tilde{r}(\bar{Y}, \bar{\eta}, \omega)$

$r_{Y}=\frac{1}{\omega}\left(\frac{\partial \tilde{r}}{\partial Y}\right)_{\bar{Y}, \bar{\eta}, \omega}$

$r_{\eta}=\frac{1}{\omega}\left(\frac{\partial \tilde{r}}{\partial \eta}\right)_{\bar{Y}, \bar{\eta}, \omega}$

$r_{Y Y}=\frac{1}{2 \omega}\left(\frac{\partial^{2} \tilde{r}}{\partial Y^{2}}\right)_{\bar{Y}, \bar{\eta}, \omega}$ 


$$
\begin{aligned}
& r_{\eta \eta}=\frac{1}{2 \omega}\left(\frac{\partial^{2} \tilde{r}}{\partial \eta^{2}}\right)_{\bar{Y}, \bar{\eta}, \omega} \\
& r_{Y \eta}=\frac{1}{\omega}\left(\frac{\partial^{2} \tilde{r}}{\partial Y \partial \eta}\right)_{\bar{Y}, \bar{\eta}, \omega}
\end{aligned}
$$

Note that the functions described in (16) are independent of $\omega$ since the reaction rate is considered linear in $\omega$. Next, the perturbed quantities are substituted into the tracer and mineral mass balances given in (10):

$$
\begin{aligned}
& \varphi \frac{\partial\left(\bar{Y}+Y^{\prime}\right)}{\partial t}+\frac{\partial}{\partial x_{i}}\left(\left(\overline{v_{i}}+v_{i}^{\prime}\right)\left(\bar{Y}+Y^{\prime}\right)\right)=\frac{\partial}{\partial x_{i}}\left(\varphi D_{i j} \frac{\partial\left(\bar{Y}+Y^{\prime}\right)}{\partial x_{i}}\right)-(1-\varphi) \alpha \psi\left(\left(\bar{Y}+Y^{\prime}\right),\left(\bar{\eta}+\eta^{\prime}\right),\left(\bar{\omega}+\omega^{\prime}\right)\right) \\
& \frac{\partial\left(\bar{\eta}+\eta^{\prime}\right)}{\partial t}=-\psi\left(\left(\bar{Y}+Y^{\prime}\right),\left(\bar{\eta}+\eta^{\prime}\right),\left(\bar{\omega}+\omega^{\prime}\right)\right) .
\end{aligned}
$$

Taking expectations of the equations in (17) and retaining up to the second order perturbations, the mean transport equations are obtained as follows:

$$
\begin{aligned}
& \varphi \frac{\partial \bar{Y}}{\partial t}+\bar{v}_{i} \frac{\partial \bar{Y}}{\partial x_{i}}+\frac{\partial\left(\overline{v_{i}^{\prime} Y^{\prime}}\right)}{\partial x_{i}}=\frac{\partial}{\partial x_{i}}\left(\varphi D_{i j} \frac{\partial \bar{Y}}{\partial x_{j}}\right)-(1-\varphi) \alpha \psi(\bar{Y}, \bar{\eta}), \\
& \frac{\partial \bar{\eta}}{\partial t}=-\psi(\bar{Y}, \bar{\eta}),
\end{aligned}
$$

Here, we define the reaction rate $\psi(\bar{Y}, \bar{\eta})$ :

$$
\psi(\bar{Y}, \bar{\eta})=\bar{\omega} r+r_{Y} \overline{\omega^{\prime} Y^{\prime}}+r_{\eta} \overline{\omega^{\prime} \eta^{\prime}}+\bar{\omega}\left(r_{Y Y} \overline{Y^{\prime 2}}+r_{\eta \eta} \overline{\eta^{2}}+r_{Y \eta} \overline{Y^{\prime} \eta^{\prime}}\right) .
$$


By subtracting the mean transport equations (18a) and (18b) from the original equations given in (17a) and (17b), respectively, and adopting the small perturbation approach, the following first order perturbation equations are obtained:

$$
\begin{aligned}
& \varphi \frac{\partial Y^{\prime}}{\partial t}+\bar{v}_{i} \frac{\partial Y^{\prime}}{\partial x_{i}}+v_{i}^{\prime} \frac{\partial \bar{Y}}{\partial x_{i}}=\frac{\partial}{\partial x_{i}}\left(\varphi D_{i j} \frac{\partial Y^{\prime}}{\partial x_{j}}\right)-(1-\varphi) \alpha\left(\omega^{\prime} r+r_{Y} \bar{\omega} Y^{\prime}+r_{\eta} \bar{\omega} \eta^{\prime}\right) \\
& \frac{\partial \eta^{\prime}}{\partial t}=-\left(\omega^{\prime} r+r_{Y} \bar{\omega} Y^{\prime}+r_{\eta} \bar{\omega} \eta^{\prime}\right)
\end{aligned}
$$

Next, a coordinate system moving with the reaction wave is introduced using the mean propagation speed $\bar{V}$ of the reaction wave:

$$
\zeta_{1}=x_{1}-\bar{V} t ; \quad \zeta_{2}=x_{2} ; \quad \zeta_{3}=x_{3} ;
$$

An expression for the wave propagation speed is determined in terms of the fluid velocity $v$ in the main flow direction in Appendix A and found that the reaction wave speed differs from the fluid flow velocity in the main direction:

$$
V=\frac{v}{\phi+\alpha(1-\phi)}
$$

(Hinch and Bhatt 1990) recognized $1 / \alpha$ as the reactant capacity number for the tracer. The latter is defined as the ratio of inlet tracer concentration to the required concentration in a pore space to transform all the minerals in the corresponding solid. The capacity number is usually less than 1.0 and, therefore, the reaction wave is 
expected to propagate slower than the fluid. In the moving coordinate system the mean equations are

$$
\begin{aligned}
& \varphi\left(\frac{\partial \bar{Y}}{\partial t}-\bar{V} \frac{\partial \bar{Y}}{\partial \zeta_{1}}\right)+v \frac{\partial \bar{Y}}{\partial \zeta_{1}}+\frac{\partial \overline{v_{i} Y^{\prime}}}{\partial \zeta_{i}}=\frac{\partial}{\partial \zeta_{i}}\left(\varphi D_{i j} \frac{\partial \bar{Y}}{\partial \zeta_{j}}\right)-(1-\varphi) \alpha \psi(\bar{Y}, \bar{\eta}), \\
& \frac{\partial \bar{\eta}}{\partial t}-\bar{V} \frac{\partial \bar{\eta}}{\partial \zeta_{1}}=-\psi(\bar{Y}, \bar{\eta})
\end{aligned}
$$

and the first-order perturbation equations are

$$
\begin{aligned}
& \varphi\left(\frac{\partial Y^{\prime}}{\partial t}-\bar{V} \frac{\partial Y^{\prime}}{\partial \zeta_{1}}\right)+v \frac{\partial Y^{\prime}}{\partial \zeta_{i}}+v_{i}^{\prime} \frac{\partial \bar{Y}}{\partial \zeta_{i}}=\frac{\partial}{\partial \zeta_{i}}\left(\varphi D_{i j} \frac{\partial Y^{\prime}}{\partial \zeta_{j}}\right)-(1-\varphi) \alpha\left(r \omega^{\prime}+r_{Y} \bar{\omega} Y^{\prime}+r_{\eta} \bar{\omega} \eta^{\prime}\right) \\
& \frac{\partial \eta^{\prime}}{\partial t}-\bar{V} \frac{\partial \eta^{\prime}}{\partial \zeta_{1}}=-\left(r \omega^{\prime}+r_{Y} \bar{\omega} Y^{\prime}+r_{\eta} \bar{\omega} \eta^{\prime} .\right)
\end{aligned}
$$

The cross-correlation term $\overline{v_{i}^{\prime} Y^{\prime}}$ in $(22 \mathrm{a})$ includes the field-scale heterogeneity effects on the mean tracer transport and represents macroscopic dispersive flux due to random variations in the fluid velocity $v_{i}$ and tracer concentration $Y$. Estimation of this and the other five reaction-related cross-correlation terms given in equation (18c) involves solving the stochastic transport equations using spectral approach described in References (Gelhar, 1993; Gelhar and Axness 1983). The perturbation quantities are assumed locally stationary in space and their spectral representation is given as the following:

$Y^{\prime}\left(\zeta_{i}, t\right)=\int_{-\infty}^{\infty} \exp \left(i k_{1}\left(\zeta_{1}+\bar{V} t\right)+i k_{2} \zeta_{2}+i k_{3} \zeta_{3}\right) d Z_{Y}\left(k_{i}, t\right)$ 
$\eta^{\prime}\left(\zeta_{i}, t\right)=\int_{-\infty}^{\infty} \exp \left(i k_{1}\left(\zeta_{1}+\bar{V} t\right)+i k_{2} \zeta_{2}+i k_{3} \zeta_{3}\right) d Z_{\eta}\left(k_{i}, t\right)$

$v_{i}^{\prime}\left(\zeta_{i}\right)=\int_{-\infty}^{\infty} \exp \left(i k_{1}\left(\zeta_{1}+\bar{V} t\right)+i k_{2} \zeta_{2}+i k_{3} \zeta_{3}\right) d Z_{v_{i}}\left(k_{i}\right)$

$\omega^{\prime}\left(\zeta_{i}\right)=\int_{-\infty}^{\infty} \exp \left(i k_{1}\left(\zeta_{1}+\bar{V} t\right)+i k_{2} \zeta_{2}+i k_{3} \zeta_{3}\right) d Z_{\omega}\left(k_{i}\right)$

Then, the perturbation equations (23a) and (23b) in the spectral domain read as:

$\varphi \frac{\partial\left(d Z_{Y}\right)}{\partial t}-\varphi \bar{V} i k_{1} d Z_{Y}+i k_{1} \nu d Z_{Y}-G_{i} d Z_{\nu_{i}}=-\varphi D_{i j} k_{i} k_{j} d Z_{Y}-(1-\varphi) \alpha\left(r d Z_{\omega}+r_{Y} \bar{\omega} d Z_{Y}+r_{\eta} \bar{\omega} d Z_{\eta}\right)$,

$\frac{\partial\left(d Z_{\eta}\right)}{\partial t}-\bar{V} i k_{1} d Z_{\eta}=-\left(r d Z_{\omega}+r_{Y} \bar{\omega} d Z_{Y}+r_{\eta} \bar{\omega} d Z\right)_{\eta}$,

Where the mean concentration gradient in (25a) is defined as $G_{i}=-\partial \bar{Y} / \partial \zeta_{i}$. Since tracer and mineral undergo a reaction process, however, the concentration perturbations change in time and therefore the transient terms as well as the mean concentration gradients cannot be neglected. The approach employed here introduces a frame of reference that follows the reaction zone not only in space but also in time, (Miralles-Wilhelm, Gelhar and Kapoor 1997). This frame of reference consists of a transformation of the time coordinate, in which, the time variations of the concentration perturbations become negligible at large times. Equations (25) can be solved by considering reaction to be the only cause of concentration perturbation variations in time. This is achieved by solving the following simplified set of equations as an intermediate step: 
$\varphi \frac{\partial\left(d Z_{Y}\right)}{\partial t}=-(1-\varphi) \alpha\left(r_{Y} \omega_{Y} d Z_{Y}+r_{\eta} \omega_{\eta} d Z_{\eta}\right)$

$\frac{\partial\left(d Z_{\eta}\right)}{\partial t}=-\left(r_{Y} \omega_{Y} d Z_{Y}+r_{\eta} \omega_{\eta} d Z_{\eta}\right)$

Hence, effective reaction coefficients $\omega_{Y}$ and $\omega_{\eta}$ are introduced for the species concentrations, which allow for the fact that $\omega$ at which concentration perturbations attenuate at the field scale may be different from the mean reaction coefficient $\bar{\omega}$ due to medium heterogeneity. The solution of this system of equations provides the following time-scaling expressions:

$d Z_{Y}=\frac{(1-\varphi) \alpha}{\varphi} d Z_{\hat{Y}} \exp \left(-\int_{0}^{t} r_{Y} \omega_{Y} d t^{\prime}\right)-\frac{(1-\varphi) \alpha}{\varphi} \int_{0}^{t} r_{\eta} \omega_{\eta} d Z_{\eta} \exp \left(-\int_{t^{\prime}}^{t} r_{Y} \omega_{Y} d t^{\prime \prime}\right) d t^{\prime}$

$d Z_{\eta}=d Z_{\hat{\eta}} \exp \left(-\int_{0}^{t} r_{\eta} \omega_{\eta} d t^{\prime}\right)-\int_{0}^{t} r_{Y} \omega_{Y} d Z_{Y} \exp \left(-\int_{t^{\prime}}^{t} r_{\eta} \omega_{\eta} d t^{\prime \prime}\right) d t^{\prime}$

Physically we can interpret the time-scaling as traveling along with the tracer amplitude as it is decreased by reaction. The result of the scaling introduces the spatial amplitudes for conservative quantities $d Z_{\hat{Y}}$ and $d Z_{\hat{\eta}}$. Following this, the equations are substituted into the perturbation equations in spectral domain. At large times, $d Z_{\hat{Y}}$ and $d Z_{\hat{\eta}}$ can be neglected. This produces two linear algebraic equations expressed in the original time coordinate for the spectral amplitudes $d Z_{Y}$ and $d Z_{\eta}$ :

$\left[i k_{1}(v-\varphi \bar{V})+(1-\varphi) \alpha r_{Y}\left(\bar{\omega}-\omega_{Y}\right)+\varphi D_{i j} k_{i} k_{j}\right] d Z_{Y}-G_{i} d Z_{v i}+(1-\varphi) \alpha r d Z_{\omega}+(1-\varphi) \alpha r_{\eta}\left(\bar{\omega}-\omega_{\eta}\right) d Z_{\eta}=0$ 
$\left[-\bar{V} i k_{1}+r_{\eta}\left(\bar{\omega}-\omega_{\eta}\right)\right] d Z \eta+r d Z_{\omega}+r_{Y}\left(\bar{\omega}-\omega_{Y}\right) d Z_{Y}=0$

The local dispersion has previously been found to have negligible influence during the evaluation of integrals of the form encountered here; therefore, it is neglected (Gelhar and Axness 1983). The expression for $d Z_{Y}$ and $d Z_{\eta}$ are then found as:

$\Delta d Z_{Y}=G_{i}\left(-i \bar{V} k_{1}+r_{\eta}\left(\bar{\omega}-\omega_{\eta}\right)\right) d Z_{\nu_{i}}+i k_{1}(1-\varphi) \bar{V} \alpha r d Z_{\omega}$

$\Delta d Z_{\eta}=-G_{i} r_{Y}\left(\bar{\omega}-\omega_{Y}\right) d Z_{v_{i}}-i k_{1} r(\nu-\phi \bar{V}) d Z_{\omega}$

with a $\Delta$ defined as

$\Delta=(v-\phi \bar{V}) k_{1}^{2} \bar{V}-i k_{1}\left[\bar{V}(1-\varphi) \alpha r_{Y}\left(\bar{\omega}-\omega_{Y}\right)-(v-\phi \bar{V}) r_{\eta}\left(\bar{\omega}-\omega_{\eta}\right)\right]$

Next, the cross correlations appearing in the mean equations need to be determined using the spectral representation theorem

$$
\overline{a^{\prime} b^{\prime}}=\int_{-\infty}^{+\infty} S_{a b} d \vec{k}=\int_{-\infty}^{+\infty} E\left[d Z_{a} * d Z_{b}^{*}\right]
$$

where $a$ and $b$ are arbitrary random fields and ' $*$ ' correspond to complex conjugate of quantity $b$. These definitions yield the cross correlations appear in our reactive flow problem as follows:

$\overline{v_{i}^{\prime} Y^{\prime}}=G_{i} I_{1}\left(v_{i}, v_{j}\right)+G_{i} r_{\eta}\left(\bar{\omega}-\omega_{\eta}\right) I_{0}\left(v_{i}, v_{j}\right)-\alpha(1-\phi) r I_{1}\left(\omega, v_{i}\right)$ 
$\overline{\omega^{\prime} Y^{\prime}}=G_{i} I_{1}\left(v_{i}, \omega\right)+G_{i} r_{\eta}\left(\bar{\omega}-\omega_{\eta}\right) I_{0}\left(v_{i}, \omega\right)-\alpha(1-\phi) r I_{1}(\omega, \omega)$

$\overline{\omega^{\prime} \eta^{\prime}}=-G_{i} r_{Y}\left(\bar{\omega}-\omega_{Y}\right) I_{0}\left(v_{i}, \omega\right)+v r I_{4}(\omega, \omega)-\phi r I_{1}(\omega, \omega)$

$\overline{Y^{\prime 2}}=0$

$\overline{\eta^{\prime 2}}=0$

$\begin{aligned} \overline{Y^{\prime} \eta^{\prime}}= & -G_{i} \alpha(1-\phi) r r_{Y}\left(\bar{\omega}-\omega_{Y}\right) I_{3}\left(v_{i}, \omega\right)+G_{i} v r I_{5}\left(\omega, v_{i}\right)+G_{i} r v r_{\eta}\left(\bar{\omega}-\omega_{\eta}\right) I_{6}\left(\omega, v_{i}\right)-\phi G_{i} r I_{2}\left(\omega, v_{i}\right) \\ & -\phi r r_{\eta}\left(\bar{\omega}-\omega_{\eta}\right) G_{i} I_{3}\left(\omega, v_{i}\right)-r^{2} v \alpha(1-\phi) I_{5}(\omega, \omega)+\phi \alpha(1-\phi) r^{2} I_{2}(\omega, \omega)\end{aligned}$

During the derivation of cross-correlations given in (31), the higher order nonlinear terms involving the product $G_{i} G_{j}$ is neglected. $I_{i}$ terms are integrals described in Appendix B. 


\section{Chapter 4}

\section{Results and Discussion}

\subsection{Field Scale Coefficients and Upscaled Reactive Flow System Dynamics}

Now that the cross correlation terms are found, equations (31), the upscaled reaction, fluid flow, and tracer velocity coefficients can be obtained explicitly from the mean equations (22). In the mean equations now there are zero-order derivatives of the reactant concentrations that are the fluctuation-induced reactive terms, first-order derivatives of the mean tracer concentration that are convective terms and secondorder derivatives of the mean tracer concentration that are dispersive terms. The following expression for the effective reaction coefficient is found by grouping terms consisting of zeroth-order derivatives of the species concentrations in the mean equations.

$\omega_{e}=\bar{\omega}-\frac{\lambda_{1} \sigma_{\omega}^{2}\left[\beta^{2}\left(\bar{\omega}-\omega_{e}\right)+\alpha(1-\phi) \bar{V}(v-\phi \bar{V}) \bar{\omega} \bar{Y} \bar{\eta}\right]}{\left(\bar{\omega}-\omega_{e}\right) \Gamma}$ 
Where $\beta$ and $\sigma_{\omega}^{2}$ are introduced as

$$
\sigma_{\omega}^{2}=b^{2} \sigma_{f}^{2}+\sigma_{\delta}^{2} ; \quad \beta=\alpha \bar{V} \bar{\eta}(1-\phi)-(v-\phi \bar{V}) \bar{Y} ; \quad \Gamma=\beta\left[\lambda_{1}\left(\bar{\omega}-\omega_{e}\right) \beta+\bar{V}(v-\phi \bar{V})\right]
$$

Setting $\bar{\omega}-\omega_{e}=\varsigma$, equation 32 simplifies to a cubic equation of the form below:

$\lambda_{1} \beta^{2} \varsigma^{3}+\beta \bar{V}(v-\phi \bar{V}) \varsigma^{2}-\lambda_{1} \sigma_{\omega}^{2} \beta^{2} \varsigma-\lambda_{1} \sigma_{\omega}^{2} \alpha(1-\phi) \bar{V}(v-\phi \bar{V}) \bar{\omega} \bar{Y} \bar{\eta}=0$

One of the roots of the cubic equation above satisfies the physics of the reaction in this problem. This root has been selected using a stability analysis and the results are shown below. 


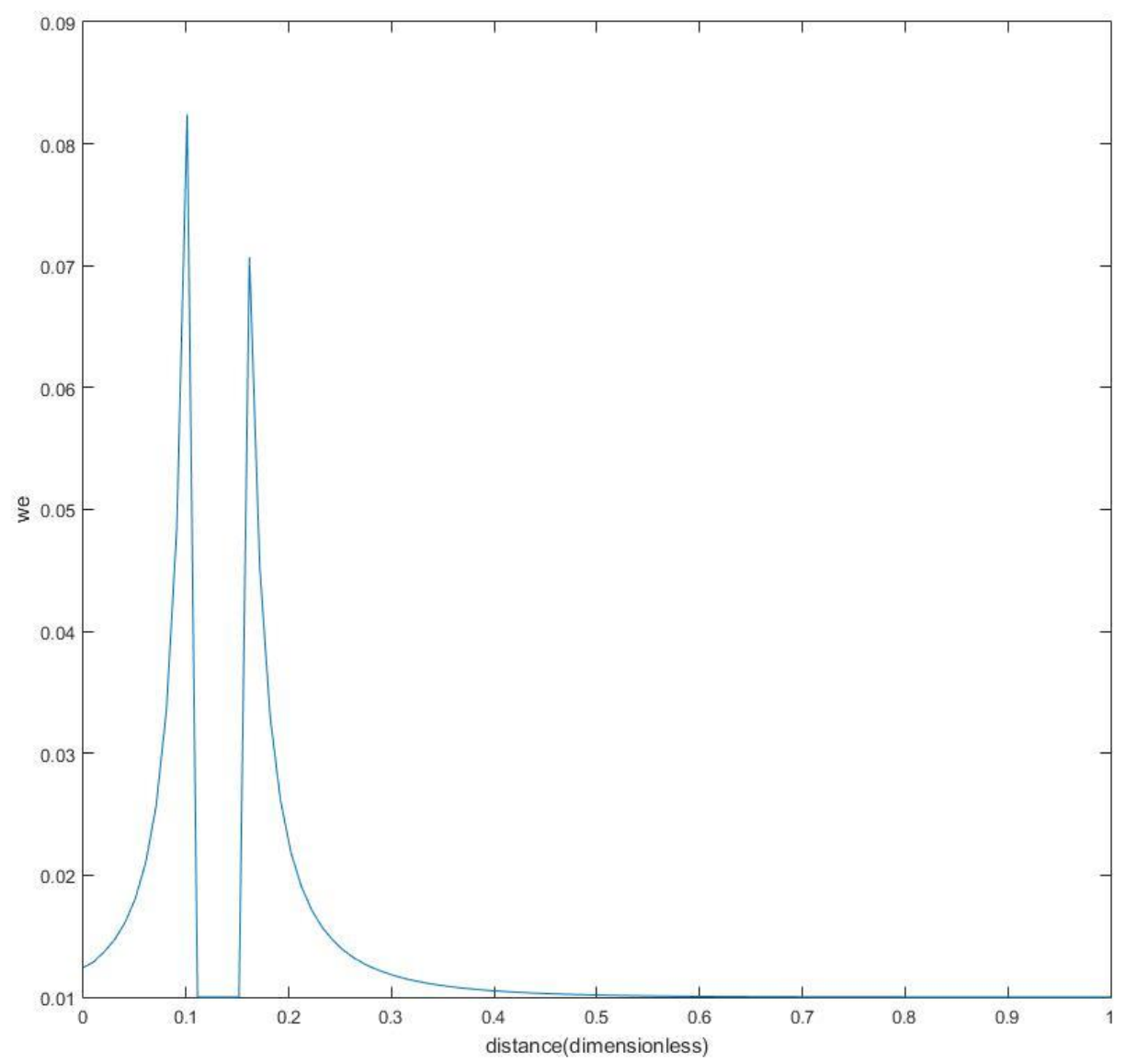

Figure 14. Effective reaction rate (we)

\section{Stability Analysis:}

The investigation starts with the effective reaction rate coefficient, which is also necessary to evaluate the other field-scale coefficients. For a fixed value of the variance and taking the model equation below,

$\lambda_{1} \beta^{2} \varsigma^{3}+\beta \bar{V}(v-\phi \bar{V}) \varsigma^{2}-\lambda_{1} \sigma_{\omega}^{2} \beta^{2} \varsigma-\lambda_{1} \sigma_{\omega}^{2} \alpha(1-\phi) \bar{V}(v-\phi \bar{V}) \bar{\omega} \bar{Y} \bar{\eta}=\frac{\partial \varsigma}{\partial t}$ 
It can be seen that all three roots converge to an integer.

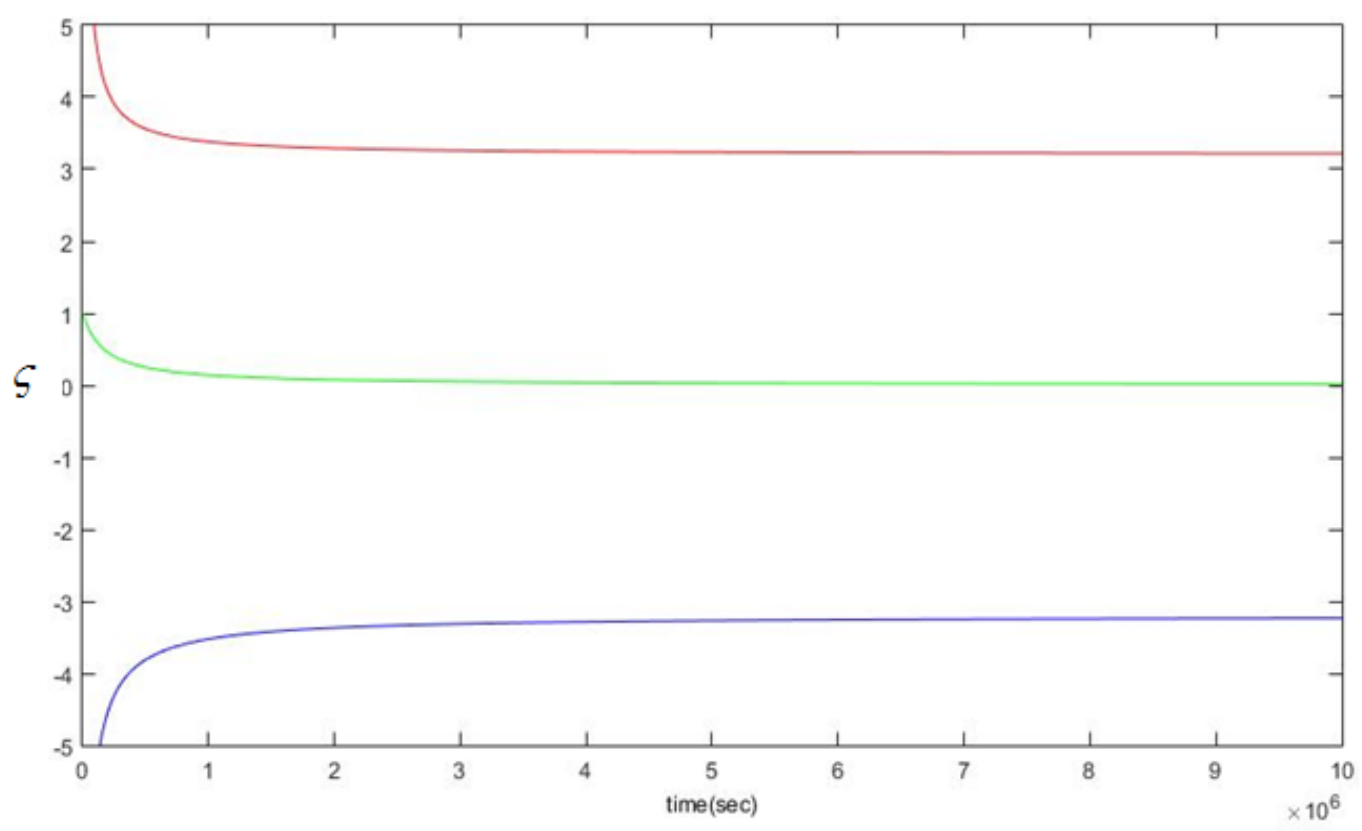

Figure 15. Stability analysis of $\varsigma$

where $\varsigma=\bar{\omega}-\omega_{e}$ indicates deviation from the mean value of the reaction rate coefficient due to the presence of heterogeneities.

These parameters play important role on the reactive flow dynamics. Similarly, the effective fluid flow velocity is determined by simply recognizing that all the advective terms (first order derivatives) in right hand side of the mean equation minus those in the left hand side is equal to $\left(v_{e}-\phi \bar{V}\right)$. This produces the following equation for effective velocity:

$v_{e}=v-\alpha(1-\phi) \bar{V}\left(\frac{A_{11}^{\prime} b \gamma \bar{\eta} \beta}{\Gamma}\right)\left\{1+\frac{\bar{\omega} \bar{Y}(v-\phi \bar{V})}{\left(\bar{\omega}-\omega_{e}\right) \beta}+\frac{\beta}{\Gamma}\left[\lambda_{1} \alpha(1-\phi) \bar{V}\left(\bar{\omega}-\omega_{e}\right) \bar{\eta}-\bar{V}(v-\phi \bar{V})\right]\right\}$ 
Where $A_{11}^{\prime}=\sigma_{f}^{2} \lambda_{1} / \gamma^{2}$ corresponds to the longitudinal dispersivity in the absence of reaction. $\gamma$ is the flow factor defined in Appendix B and taken to be equal to $\gamma=\exp \left(\sigma_{f}^{2} / 6\right)$. The graph below shows the result for equation (33). Considering the average linear velocity considered for the homogeneous case, 10E-3, it could be concluded that the heterogeneity has a damping effect on linear velocity of the acid slightly after the injection point.

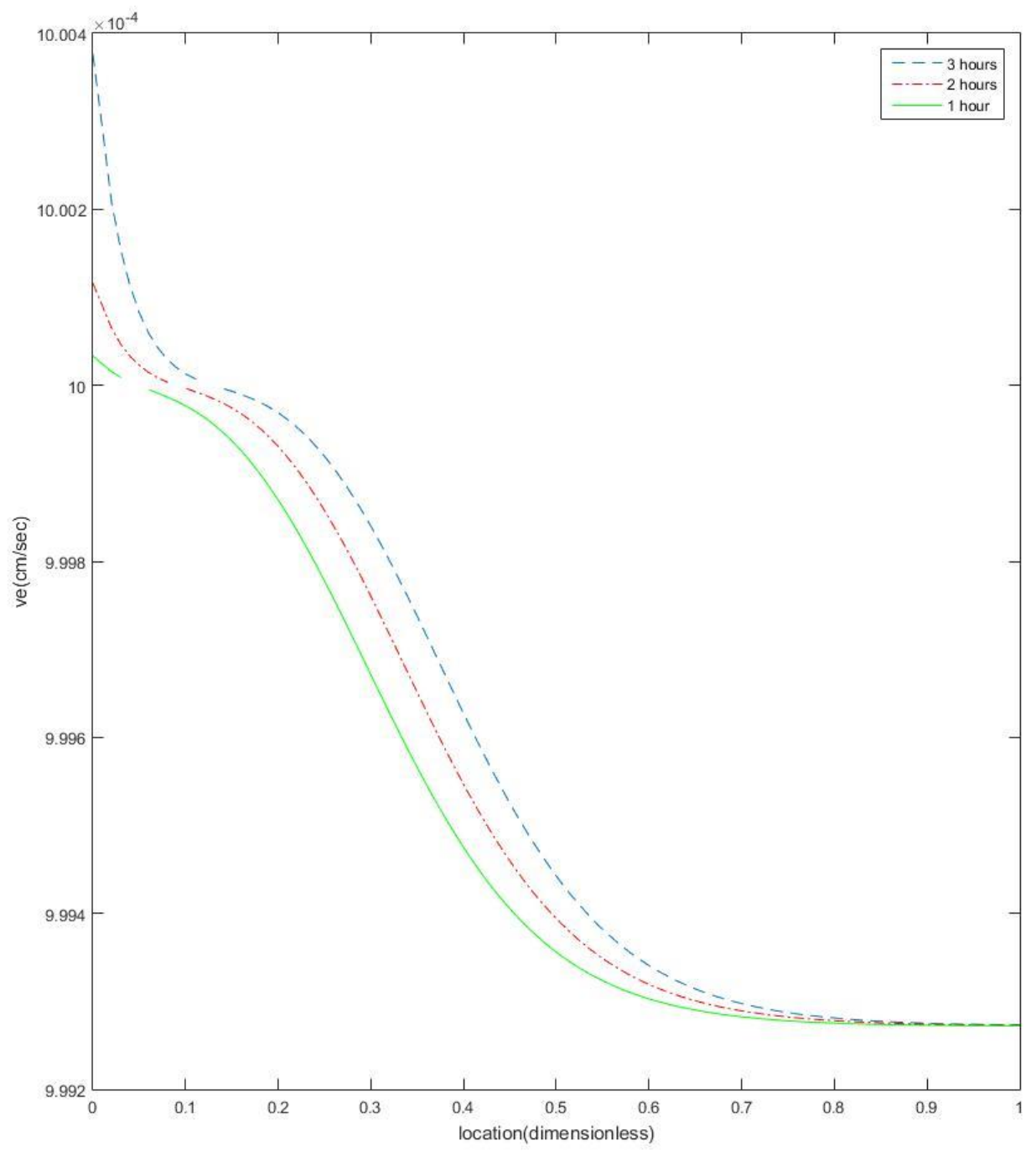

Figure 16. Effect of time on the Effective linear velocity (ve) 
The figures below show the effect of permeability variance and correlation length on effective linear velocity

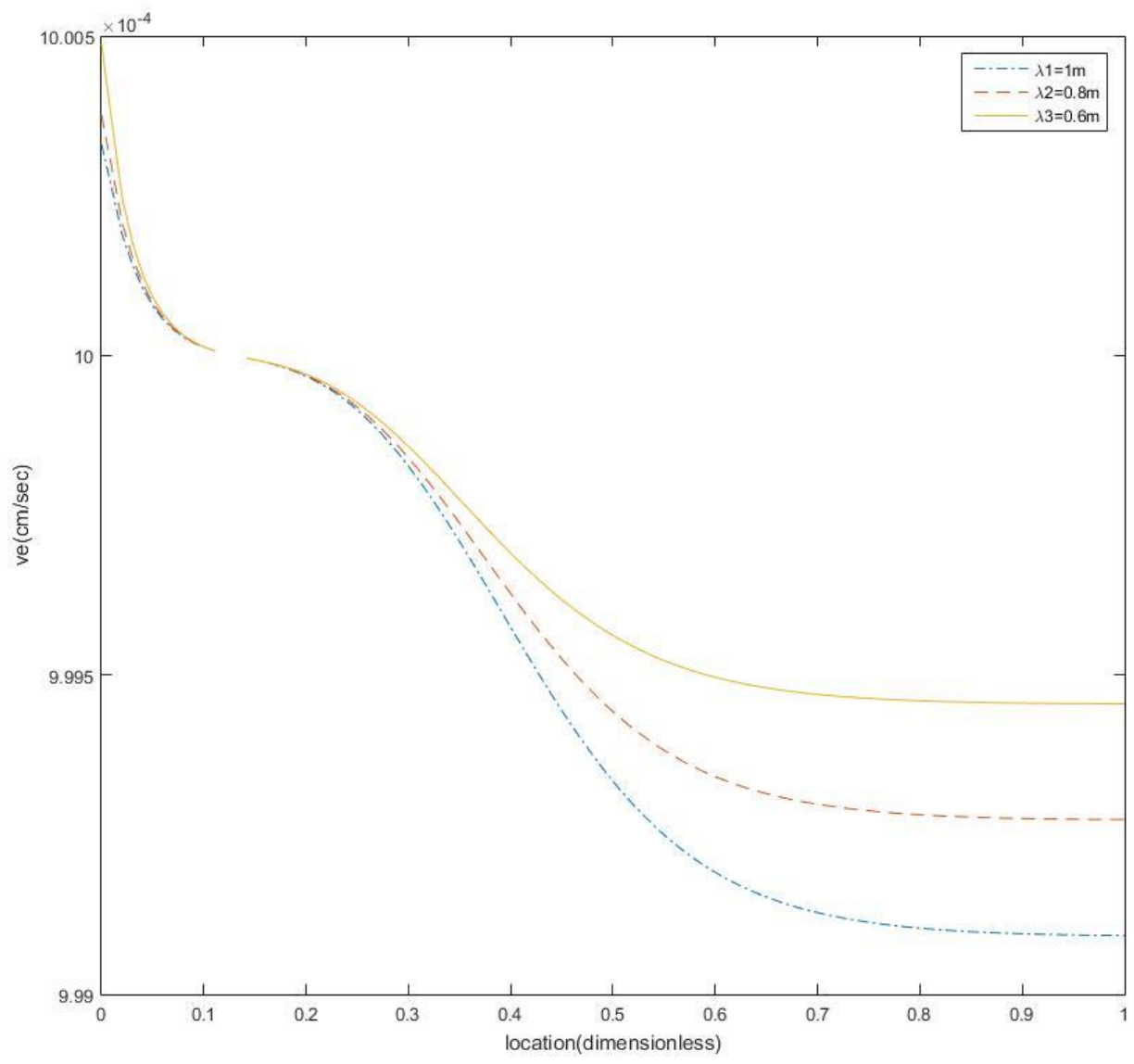

Figure 17. Effect of permeability correlation length on the Effective linear velocity (ve) 


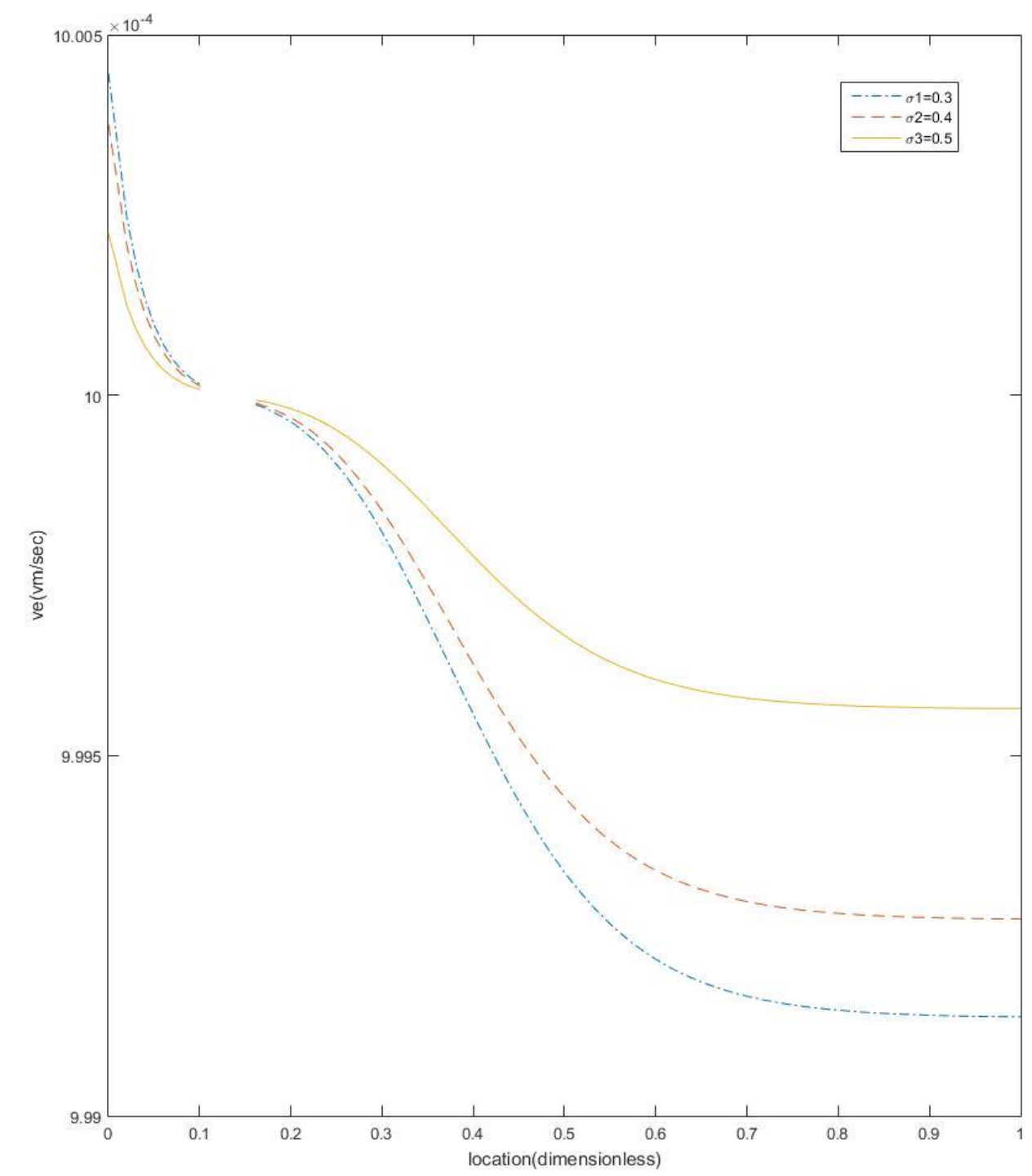

Figure 18. Effect of permeability variance on the Effective linear velocity (ve) 
Finally, the longitudinal dispersion coefficient $v A_{11}$ could easily be obtained by grouping together the dispersive terms in the mean tracer mass balance into the $\partial\left(v A_{11} \partial \bar{Y} / \partial \zeta_{1}\right) / \partial \zeta_{1}$ form. Hence, the longitudinal effective dispersivity is obtained:

$$
A_{11}=A_{11}^{\prime}\left[\frac{v \alpha(1-\phi) \bar{V}^{2} \bar{\eta}}{\Gamma}\right]
$$

The following graph is depicts the results of the longitudinal effective dispersivity in equation (34) for three different time steps. 


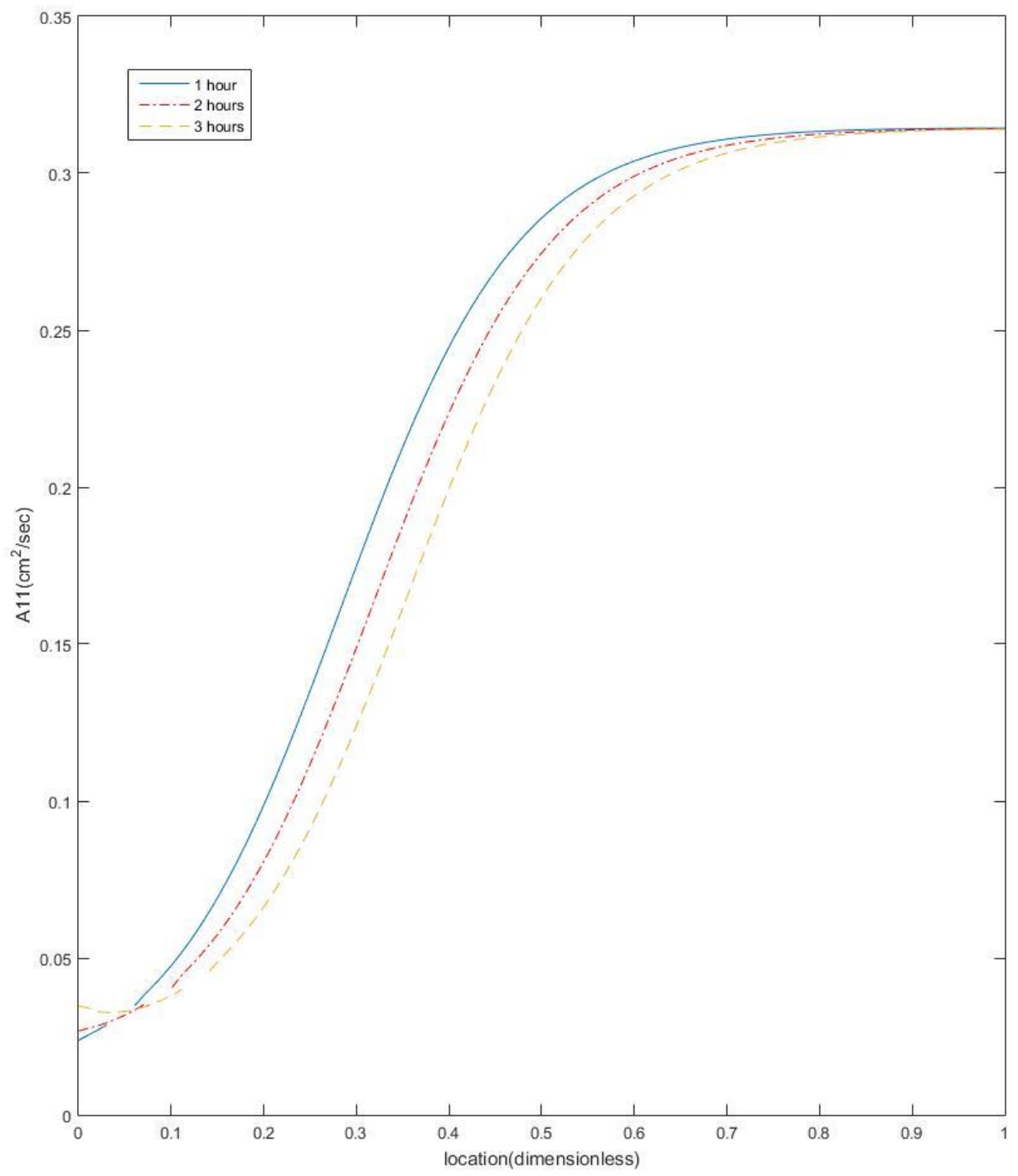

Figure 19. Effect of time on the longitudinal effective dispersivity (A11)

Next, the effect of permeability variance and correlation length on the effective longitudinal macrodispersivity is shown. 


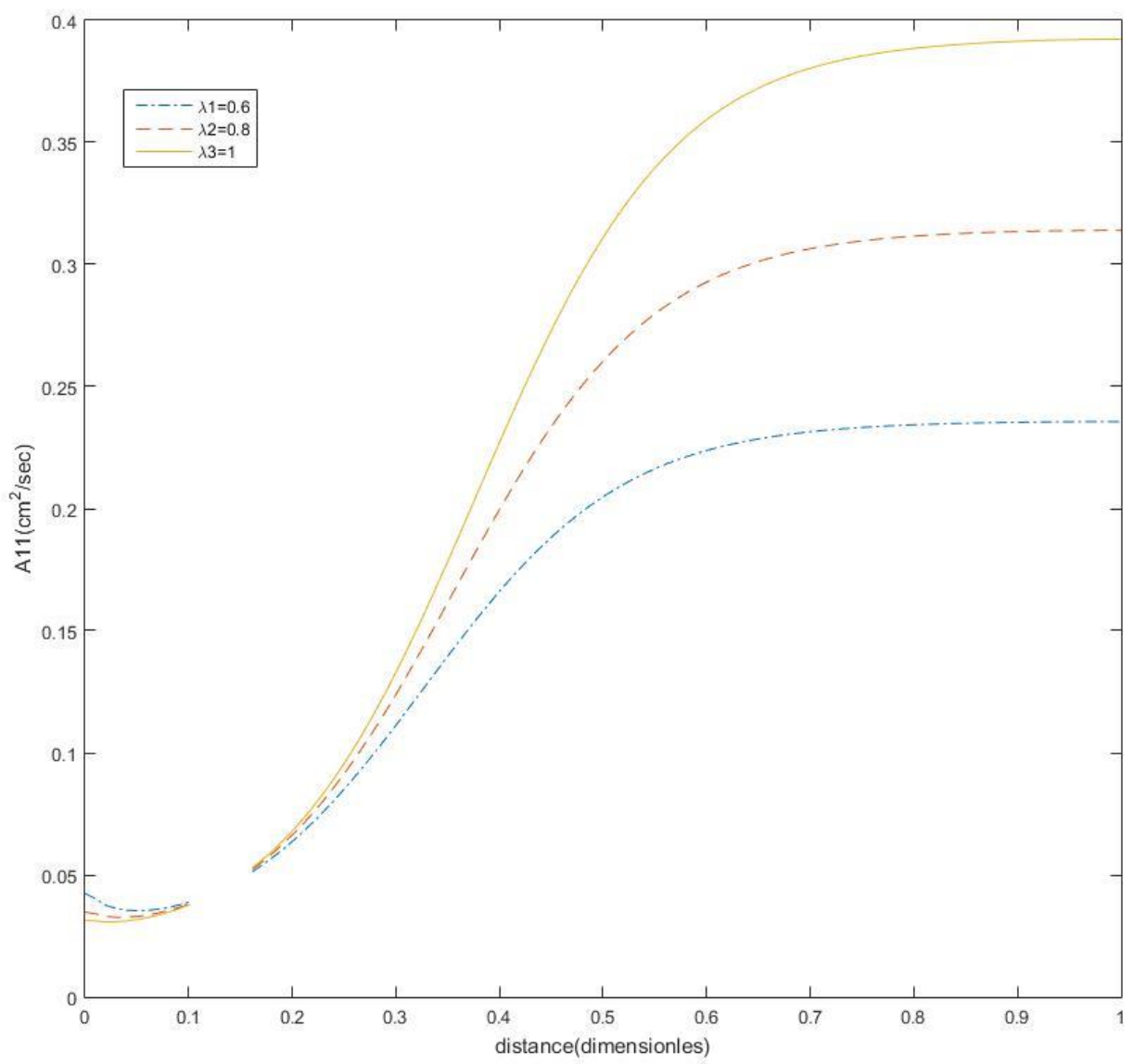

Figure 20. Effect of permeability correlation length on effective dispersivity (A11) 


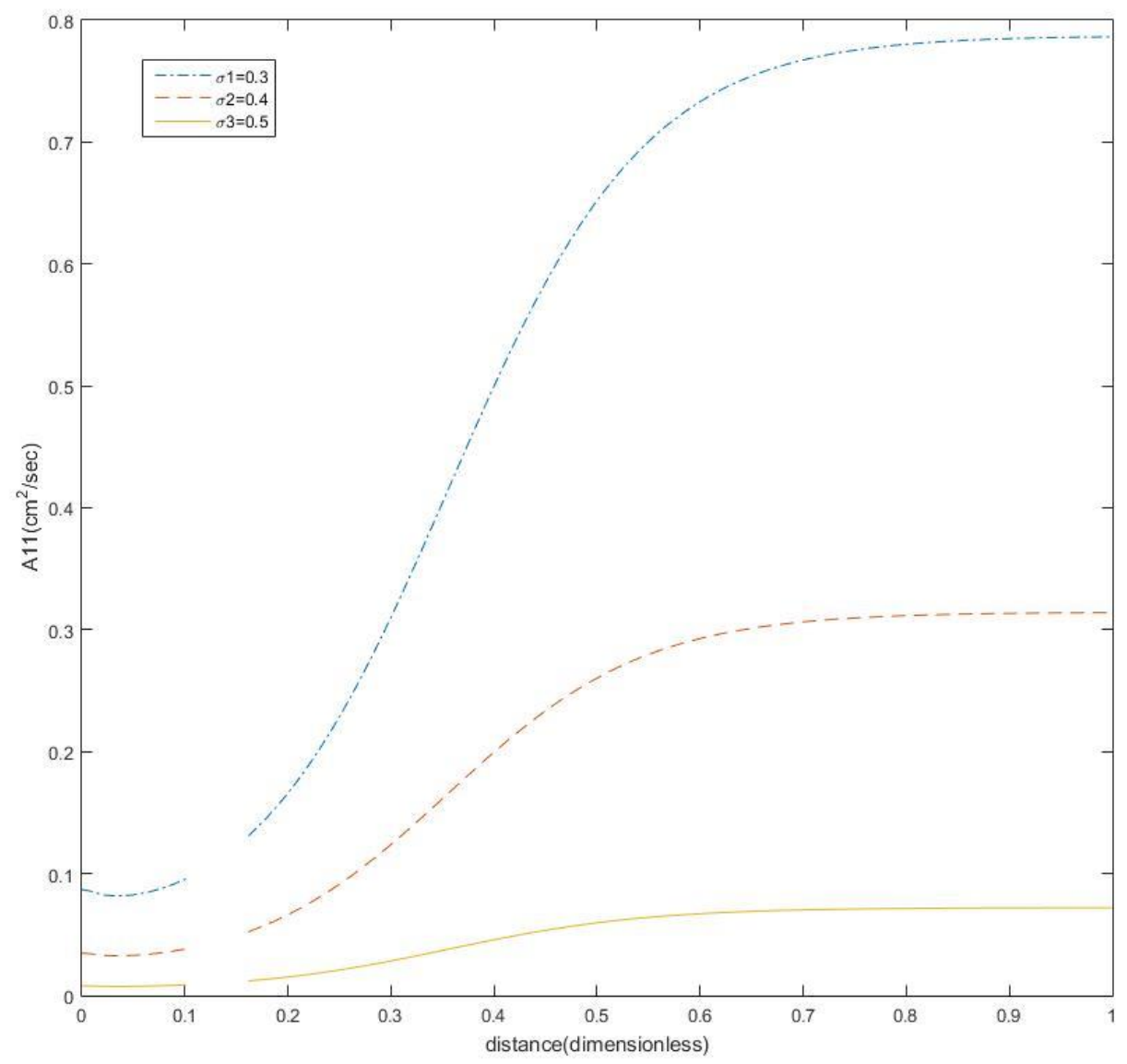

Figure 21. Effect of permeability variance on the effective dispersivity (A11)

Having the new upscaled parameters substituted in the governing equation of the flow, the field-scale partial differential equations for the tracer and mineral mass are written as below:

$\phi \frac{\partial \bar{Y}}{\partial t}+v_{e} \frac{\partial \bar{Y}}{\partial x_{1}}=\frac{\partial}{\partial x_{1}}\left(\phi v A_{11} \frac{\partial \bar{Y}}{\partial x_{1}}\right)-\alpha(1-\phi) \omega_{e} \bar{Y} \bar{\eta}+\ldots$

$\frac{\partial \bar{\eta}}{\partial t}=-\omega_{e} \bar{Y} \bar{\eta}+\ldots$ 
Here, it is important to note that $v A_{11}$ product represents the upscaled dispersive mixing due to non-uniform advection only, since the local dispersion has been neglected during the application of spectral theory. The representative elementary values are set for the parameters of the upscaled governing equations and the coupled equations are solved using an implicit finite difference method in MATLAB. The results below show that the heterogeneity has significant effect on the mineral concentration at the injection point and the acid concentration at the point far downstream.

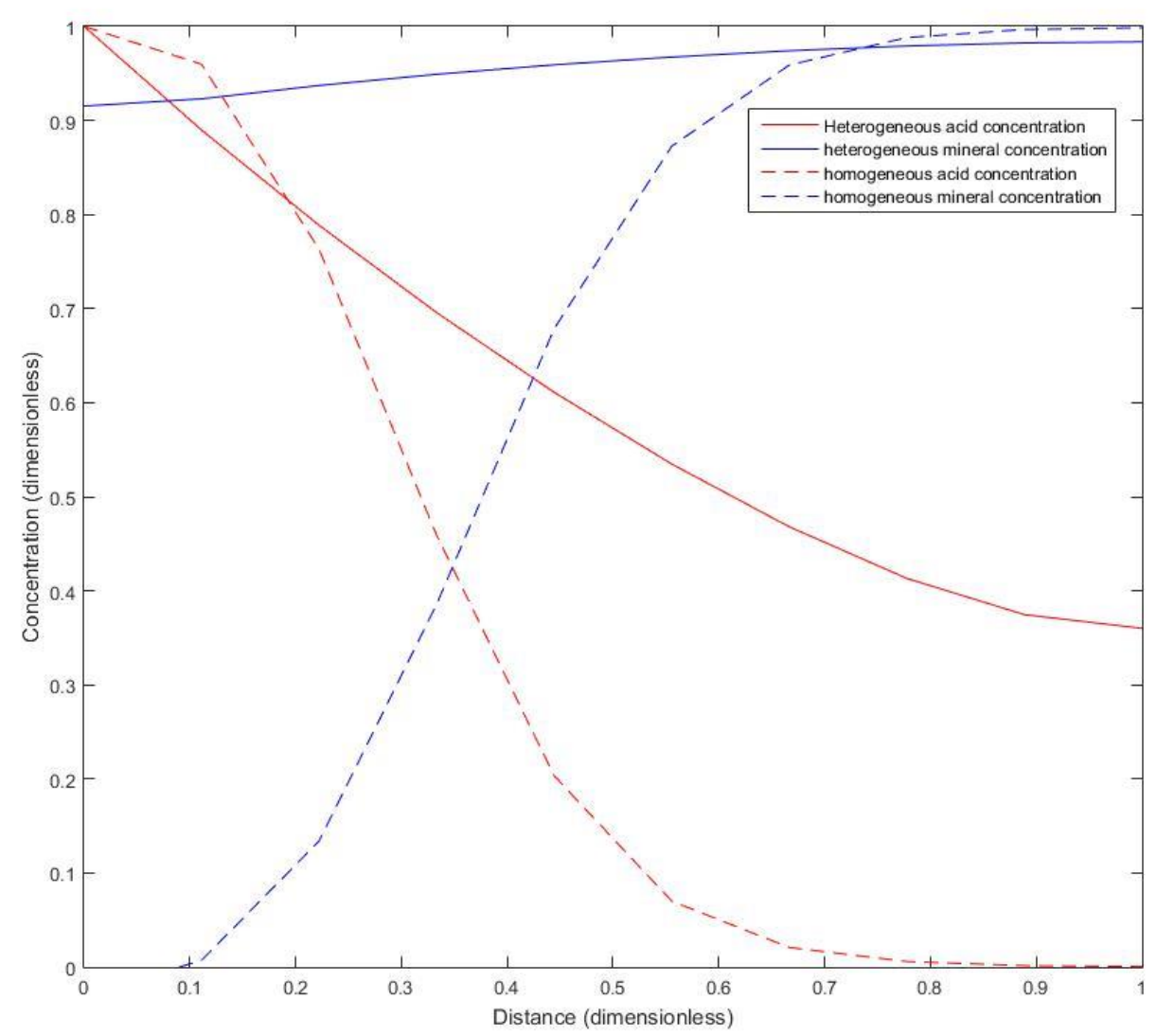

Figure 22. Acid and mineral concentration in both Homogeneous and Heterogeneous cases 


\section{Chapter 5}

\section{Concluding Remarks}

In this study an analytical approach based on small perturbation theory is used to upscale the heterogeneity effect of porous medium permeability on fluid flow, transport and dissolution reaction. The upscaled governing equations (eqns. 32-35) show rich nonlinear interplay between the existing mechanisms and includes the local characteristics of formation heterogeneity in permeability, i.e., variance and correlation length of the permeability. Increasing the correlation length of the permeability leads to higher upscaled velocity and reaction rates that resembles possibility of non-uniform reaction wave propagation in the heterogeneous medium, i.e., fluid fingering phenomena. Increasing the variance of permeability however leads to more macro dispersion effect. The upscaled governing equations can be used as a proxy to significantly decrease the simulation time associated with field application of acidizing while keeping the complexity of the problem.

Upscaling of the governing equations was performed based on the assumption of an ideal liquid solution where the mineral dissolution does not impact the fluid 
properties such as viscosity and density. This needs to be further investigated including the viscosity and density of the fluid as stochastic random parameter. 


\section{Bibliography}

Achanta, S., J. H. Cushman, and M.R. Okos. "On multicomponent, multiphase thermomechanics with interfaces." Int. J. Eng. Sci. 32 (1994): 1717-1738.

Aris, R. "On the dispersion of a sploute in a fluid flowing through a tube." Proc. Royal Soc. London 235 (1956): 67-77.

Bennethum, Lynn Schreyer, and John Cushman. "Multiscale, hybrid mixture theory for swelling systems. I. Balance laws." Internat. J. Engrg. Sci. 34, no. 2 (1996): 125-145.

Bensoussan, A., J.L. Lions, and G.C Papanicolaou. "Asymptotic analysis for periiodic structures." North-Holland, 1978.

Berkowitz, Brian, Andrea Cortis, Marco Dentz, and Harvey Scher. "Modeling Non-Fickian Transport in Geological formations as a Continuous Time Random Walk." Reviews of Geophysics 44, no. 2 (2006).

Berner, H. "A general theory of Taylor dispersion phenomena." Physico-Chemical Hydrodynamics 1 (1980): 91-123.

Bhattacharya, RN, and UK Gupta. "Application of central limit theorems to solute transport in saturated porous media: From kinetic to field scales." Dynamics of Fluids in Hierarchical Porous, 1990.

Brenner, H. "Dispersion resulting from flows through spatially periodic porous media." Phil. Trans. Roy. Soc. Lond. 297 (1980): 81-133.

Coleman, B. D., and W. Noll. "The thermodynamics of elastic materials with heat conduction and viscosity." Archive for Rational Mechanics Analysis 13, no. 1 (1963): 167-178.

Darcy, Henry. "Les fontaines publiques de la ville de Dijon." Victor Dalmont, 1856.

De Giorgi, E. "G-operators and $\Gamma$-convergence." Proceedings of the International Congress of Mathematicians Warsazwa. Amsterdam: PWN Polish Scientific Publishers and North-Holland, 1984. 1175-1191.

Durlofsky, Louis J., Richard C. Jones, and William J. Milliken. "A Nonuniform Coarsening Approach for the Scale-up of displacement Processes in Heterogeneous Porous Media." Advances in Water Resources 20, no. 5-6 (1997): 335-347.

Fan, Yong, Yuqin Wang, and R.S. Bryant. "A Comprehensive Model of Matrix Acidization." SPE European Formation Damage Conference. The Hague, The NEtherlands: SPE, 1997.

Fathi, Ebrahim, I.Y. Akkutlu, and L.B. Cunha. "Effects of Porosity and Material Fluctuations on Gas Transport and Sorption Kinetics in Coalbeds." Canadian International Petroleum Conference. Calgary, 2007. 
Fogler, H.S, K. Lund, and C.C. McCune. "Predicting the Flow and Reaction of HCL/HF Acid Mixtures in Porous Sandstone Cores." Society of Petroleum Engineers Journal, 1976: 248-260.

Gavrilenko, P., and Y. Guéguen. "Flow in fractured media: A modified renormalization method." Water Resources Research , 1998.

Gelhar, Lynn W. Stochastic Subsurface hydrology. Englewood Cliffs, N.J., U.S.A: PrenticeHall, 1993.

Gelhar, Lynn W., and Carl L. Axness. "Three dimesnional Analysis of Macrodispersion in Aquifers." Water Resources Research 19, no. 1 (1983): 161-180.

Hassanizadeh, S. Majid, and W.G. Gray. "General Conservation Equations for Multi-phase Systems 1.Averaging Procedure." Advances in Water Resources 2 (1979): 131-144.

Hinch, E.J., and B.S. Bhatt. "Stability of an Acid Moving Through Porous Rock." Journal of Fluid Mechanic 212 (1990): 279-288.

King, P.R. "The use of renormalization for calculatig effective permeability." Transport in Porous media 4 (1989): 37-58.

Kozlov S. "Averaging random structures." Soviet Math. Dokl. 19 (1978).

Miralles-Wilhelm, F., Lynn W. Gelhar, and V. Kapoor. "Stochastic Analysis of Oxygenlimited Biodegradation in Three-dimensionally Heterogeneous Aquifers." Water Resources Research 33 (1997): 1251-1263.

Montroll, Elliott, and George Weiss. "Random Walks on Lattices. II." Journal of Mathematical Physics 6, no. 2 (1965).

Murat, F. "H-Convergence." Seminaire dAnalyse Fonctionelle et Numerique de lUniversite dAlger. 1977.

Noetinger, B., and T. Estebenet. "Up-scaling of double porosity fractured media using continuous-time random walks methods." Transport in Porous media 39 (2000): 315337.

Sablok, R., and Khalil Aziz. "Upscaling and Discretization Errors in Reservoir Simulation." Petroleum Science and Technology 26, no. 10-11 (2008): 1161-1186.

Sherwood, J.D. "Stability of a Plane Reaction Front in a Porous Medium." Chemical Engineering Science 42 (1987): 1823-1829.

Steefel, C.I., D.J. DePaolo, and P.C. Lichtner. "Reactive Transport Modeling: An essential tool and a new research approach for the Earth Sciences." Earth and Planetary Sciences Letters 240, no. 3-4 (2005): 539-558.

Tartar, L. "Cours Peccot au Coll’ege de France." Paris, 1977.

Warren, J. E., and H. S. Price. "Flow in Heterogeneous Porous Media." SPE Journal 1 (1961): 153-169. 
Welty, Claire, and Lynn W. Gelhar. "Stochastic Analysis of the Effects of Fluid Density and Viscosity Variability on Macrodispersio in Heterogeneous Porous Media." Water Resources Research 27 (1991): 2061-2075.

Whitaker, S. "Diffusion and dispersion in porous media." Am. Inst. Chem. Engrs. J. 13 (1967): 420-427.

Whitaker, Stephen. The method of volume averaging. Kluwer Academic Publishers, 1999.

Wilson, Kenneth G. "Renormalization Group and Critical Phenomena. I. Renormalization Group and the Kadanoff Scaling Picture." Physical Review B 4, no. 9 (1971): 31743183.

Y., Fan, Wang Y., and Bryant R.S. "A comprehensive Model of Matrix Acidiziation." Paper presented at the SPE European Formation Damage Conference. The Hague, The Netherlands, 1997.

Zhang, Dongxiao, Roko Andricevic, Alexander Sun, Xiaolong Hu, and Guowei He, "Solute flux approach to transport through spatially nonstationary flow in porous media." Water Resources Research 36, no. 8 (2000): 2107-2120. 


\section{Appendix A - Estimation of the Reaction Wave Propagation Velocity}

Now that we know the reaction is steadily moving we need to find a solution using the reaction wave approach. Propagation speed of the reaction front is $V$ as the injecting fluid flows along the $\zeta_{1}$ direction at a speed $v$. The solution is as followed:

$Y=Y_{0}\left(\zeta_{1}-V t\right)$

$\eta=\eta_{0}\left(\zeta_{1}-V t\right)$

The transient effects and local diffusion are neglected and the the governing equations become

$-\phi V \frac{\partial Y}{\partial \zeta_{1}}+v \frac{\partial Y}{\partial \zeta_{1}}=-\alpha(1-\phi) \tilde{r}=-\alpha(1-\phi) V \frac{\partial \eta}{\partial \zeta_{1}}$

At the injecting point the acid is being injected with a constant rate and the concentration equals to inlet concentration, the mineral is completely untouched and stationary; whereas far downstream, all of the acid is spent and no mineral is reacted:

$$
\begin{array}{lll}
Y \rightarrow 0 ; & \eta \rightarrow 1 ; & \zeta_{1} \rightarrow-\infty \\
& & \\
Y \rightarrow 1 ; & \eta \rightarrow 0 ; & \zeta_{1} \rightarrow+\infty
\end{array}
$$

Integrating equation (B.2) once and using the upstream condition, we obtain 


$$
(v-\phi V)(Y-1)=-\alpha(1-\phi) V \eta
$$

Applying downstream condition, we find

$$
v-\phi V=\alpha V(1-\phi)
$$

which finally gives the reaction front velocity as

$$
V=\frac{v}{\phi+\alpha(1-\phi)}
$$




\section{Appendix B - Cross- correlation Integrals,}

To find the cross correlations terms we first need to evaluate the integrals $I_{0}$ to $I_{6}$, which are solved by the stratified medium approximation brought in (Gelhar and Axness 1983). The expression for $I_{0}$ is:

$I_{0}(f, f)=\int_{-\infty}^{+\infty} \frac{S_{f f}}{\Delta} d \vec{k}$

The complex conjugate of $\Delta$ is found and multiplied to the integral argument

$I_{0}(f, f)=\int_{-\infty}^{+\infty} \frac{\Delta^{*} S_{f f}}{\Delta \Delta^{*}} d \vec{k}=\frac{1}{\bar{V}(v-\phi \bar{V})} \int_{-\infty}^{+\infty} \frac{S_{f f}}{k_{1}^{2}+\left(\frac{E}{\bar{V}}\right)^{2}} d \vec{k}$

where

$E=\frac{\left(\bar{\omega}-\omega_{Y}\right) \bar{V} \alpha(1-\phi) r_{Y}-(v-\phi \bar{V}) r_{\eta}\left(\bar{\omega}-\omega_{\eta}\right)}{v-\phi \bar{V}}$

The integration of the imaginary terms in the nominator are zero because the denominator of the integral in (B2) is an even function of $k_{1}$. Multiplying both numerator and denominator with $\lambda_{1}$ we obtain 


$$
I_{0}(f, f)=\frac{\lambda_{1}^{2}}{\bar{V}(v-\phi \bar{V})} \int_{-\infty}^{+\infty} \frac{S_{f f} d \vec{k}}{\left[\lambda_{1}^{2} k_{1}^{2}+\left(\frac{\lambda_{1} E}{\bar{V}}\right)^{2}\right]}
$$

where

$S_{f f} d \vec{k}=\frac{\sigma_{f}^{2} \lambda_{1} \lambda_{2} \lambda_{3} d k_{1} d k_{2} d k_{3}}{\pi^{2}\left[1+\left(\lambda_{1} k_{1}\right)^{2}+\left(\lambda_{2} k_{2}\right)^{2}+\left(\lambda_{3} k_{3}\right)^{2}\right]^{2}}$.

Introducing variables $u_{i}=\lambda_{i} k_{i} \mathrm{i}=1,2,3$ and defining $\kappa=\lambda_{1} E / \bar{V}$

$$
I_{0}(f, f)=\frac{\lambda_{1}^{2} \sigma_{f}^{2}}{\bar{V}(v-\phi \bar{V}) \pi^{2}} \int_{-\infty}^{+\infty} \frac{d \vec{u}}{\left(u_{1}^{2}+\kappa^{2}\right)+\left(1+\vec{u}^{2}\right)}
$$

The integral in this form can be evaluated in spherical coordinates to produce the following result

$$
I_{0}(f, f)=\frac{\lambda_{1}^{2} \sigma_{f}^{2}}{\bar{V}(v-\phi \bar{V})}\left(\frac{1}{\kappa}-\frac{1}{1+\kappa}\right)
$$

The integrals $I_{1}$ to $I_{6}$ are also evaluated using the same approach. The results are below

$$
\begin{aligned}
& I_{1}(f, f)=\frac{-\lambda_{1} \sigma_{f}^{2}}{(v-\phi \bar{V})}\left(\frac{1}{1+\kappa}\right) \\
& I_{2}(f, f)=\frac{\lambda_{1}^{2} \sigma_{f}^{2}}{(v-\phi \bar{V})^{2}}\left(\frac{1}{\kappa}-\frac{1}{1+\kappa}\right)
\end{aligned}
$$




$$
\begin{aligned}
& I_{3}(f, f)=0 \\
& I_{4}(f, f)=\frac{-\lambda_{1} \sigma_{f}^{2}}{\bar{V}(v-\phi \bar{V})}\left(\frac{1}{1+\kappa}\right) \\
& I_{5}(f, f)=\frac{\lambda_{1}^{2} \sigma_{f}^{2}}{\bar{V}(v-\phi \bar{V})^{2}}\left(\frac{1}{\kappa}-\frac{1}{1+\kappa}\right) \\
& I_{6}(f, f)=0
\end{aligned}
$$

For the stratified medium approximation, the spectra for cross-correlations can be related to the spectrum of random permeability filed:

$$
S_{\vartheta \bigcup_{11}}=\frac{v^{2}}{\gamma^{2}} S_{f f}, \quad S_{\vartheta \mid \omega}=\frac{b}{\gamma} S_{f f}, \quad S_{\omega \omega}=b^{2} S_{f f}+S_{\delta \delta}, \quad \gamma=\frac{\mu \phi v}{k J_{1}}, \quad J_{1}=-\frac{\partial p}{\partial x_{1}}
$$

Here, partial correlation residuals $\delta_{i}, i=1,2,3$ are assumed to be statistically homogeneous random fields and described by three dimensional anisotropic autocovariance with the following spectrum

$$
S_{\delta \delta}=\frac{\sigma_{f}^{2} \lambda_{1} \lambda_{2} \lambda_{3}}{\pi^{2}\left[1+\left(\lambda_{1} k_{1}\right)^{2}+\left(\lambda_{2} k_{2}\right)^{2}+\left(\lambda_{3} k_{3}\right)^{2}\right]^{2}}
$$


Table 2. Parameters of Advection-Reaction Problem in Heterogeneous Porous Media

\begin{tabular}{|l|l|}
\hline Parameter & Value \\
\hline$\varnothing$ & 0.25 \\
\hline$v$ & 0.001 \\
\hline$\alpha$ & 0.01 \\
\hline$\sigma_{\lambda}$ & 0.01 \\
\hline $\bar{\omega}$ & \\
\hline$\sigma_{f}$ & \\
\hline
\end{tabular}

